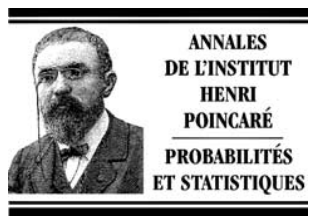

www.elsevier.com/locate/anihpb

\title{
Markovian bridges and reversible diffusion processes with jumps
}

\author{
Nicolas Privault ${ }^{\text {a }}$, Jean-Claude Zambrini ${ }^{\text {b }}$ \\ a Département de mathématiques, université de La Rochelle, avenue Michel Crépeau, 17042 La Rochelle, France \\ ${ }^{\mathrm{b}}$ Grupo de Física Matemática, Universidade de Lisboa, Avenida Prof. Gama Pinto 2, 1649-003 Lisboa, Portugal
}

Received 12 September 2002; received in revised form 18 August 2003; accepted 25 October 2003

Available online 12 May 2004

\begin{abstract}
Markovian bridges driven by Lévy processes are constructed from the data of an initial and a final distribution, as particular cases of a family of time reversible diffusions with jumps. In this way we construct a large class of not necessarily continuous Markovian Bernstein processes. These processes are also characterized using the theory of stochastic control for jump processes. Our construction is motivated by Euclidean quantum mechanics in momentum representation, but the resulting class of processes is much bigger than the one needed for this purpose. A large collection of examples is included.
\end{abstract}

(c) 2004 Elsevier SAS. All rights reserved.

\section{Résumé}

Des ponts markoviens dirigés par des processus de Lévy sont construits, étant données leurs distributions initiale et finale, comme cas particuliers d'une classe de diffusions réversibles avec sauts. On obtient ainsi une famille de processus de Bernstein markoviens non nécessairement continus, qui peuvent être caractérisés à l'aide du contrôle stochastique pour les processus de sauts. Notre construction est motivée par la mécanique quantique euclidienne en représentation d'impulsion, mais la famille de processus ainsi obtenue est plus vaste que celle nécessitée par cette application. Différents exemples sont présentés.

(c) 2004 Elsevier SAS. All rights reserved.

MSC: 60J25; 81S20; 47D07

Keywords: Lévy processes; Bridges; Time reversal; Euclidean quantum mechanics

Mots-clés : Processus de Lévy ; Retournement du temps ; Mécanique quantique euclidienne

\section{Introduction}

Euclidean quantum mechanics yields a probabilistic approach to Schrödinger equations, which relies on the construction of time reversible stochastic processes. A probabilistic counterpart of a quantum system with

E-mail addresses: nprivaul@univ-lr.fr (N. Privault), zambrini@ cii.fc.ul.pt (J.-C. Zambrini). 
symmetric (more precisely, self-adjoint) Hamiltonian $H$ is provided by considering positive solutions of two heat equations which are adjoint with respect to the time parameter:

$$
-\hbar \frac{\partial \eta_{t}^{*}}{\partial t}(q)=H \eta_{t}^{*}(q) \quad \text { and } \quad \hbar \frac{\partial \eta_{t}}{\partial t}(q)=H \eta_{t}(q), \quad t \in[r, v], q \in \mathbb{R}^{d},
$$

where $\hbar$ is Planck's constant (afterwards set equal to 1 in this paper) and $[r, v]$ is a fixed interval, and by postulating that the density of the law at time $t$ of the system is given by the product $\eta_{t}(q) \eta_{t}^{*}(q)$, instead of the product of the solution of Schrödinger's equation with its complex conjugate. This approach allows moreover to construct time reversible diffusion processes which precisely have the law $\eta_{t}(q) \eta_{t}^{*}(q) d q$ at time $t$, see $[31,9,2]$ when the Hamiltonian is a self-adjoint Schrödinger operator of the form $H=-\frac{\hbar^{2}}{2} \Delta+V(q)$ and $V$ is a scalar potential in Kato's class. We refer the reader to [8] for a detailed survey of the relations between this method, and Feynman's path integral approach to quantum mechanics, when the processes have continuous trajectories.

In this paper we generalize this construction to the case where the above Schrödinger operator is replaced by a pseudo-differential operator. Our motivations are twofold. First, the study of the probabilistic counterpart of quantum mechanics in the momentum representation and its relation with the one of the position representation, the link between these representations being given by the Fourier transform which maps position operators to momentum operators, and scalar potentials to pseudo-differential operators. This illustrates the more general aim of this program of construction of quantum-like reversible measures. They provide (through their Hilbert space analytical models) fresh structural relations between stochastic processes generally regarded as unrelated in probability theory. Our second motivation is to treat relativistic Hamiltonians along the line of [19], but in a time reversible framework.

Lévy bridges have been studied and constructed by several authors, see e.g. [14] and Section VIII.3 in [4]. However, an absolute continuity condition with respect to Lebesgue measure is generally imposed on the law of the process, thus excluding simple Poisson bridges and many other more complex processes. Our construction of reversible diffusions with jumps provides, in particular, a general construction of Markovian bridges with given initial and final distributions $\pi_{r}$ and $\pi_{v}$. For this we use a result of Beurling [5] which, under the assumption of existence of densities with respect to a fixed reference measure, asserts the existence of initial and final conditions $\eta_{r}$ and $\eta_{v}^{*}$ for (1.1) such that $\pi_{r}=\eta_{r} \eta_{r}^{*}$ and $\pi_{v}=\eta_{v} \eta_{v}^{*}$. In the case of Dirac measures as initial and final laws, the processes obtained in this way are bridges in the usual sense of conditioning a Lévy process $\left(\xi_{t}\right)_{t \in[r, v]}$ with the values $\xi_{r}=a$ and $\xi_{v}=b$. In particular we construct forward and backward Lévy processes with Dirac measures as initial and final laws. In this case we extend existing results on the martingale representation of time-reversed processes, cf. e.g. [23]. We also show how time reversible processes can be constructed from non-symmetric Lévy processes and generators.

We use the term "bridge" in the wide sense, i.e. a process which is determined from initial and final laws (not necessarily Dirac measures) will be called a bridge. Bridges and more generally diffusions with jumps, reversible on $[r, v]$, are constructed via the forward and backward Markov transition semi-groups

$$
p(t, k, u, d l)=\frac{\eta_{u}(l)}{\eta_{t}(k)} h(t, k, u, d l), \quad \text { and } \quad p^{*}(s, d j, t, k)=\frac{\eta_{s}^{*}(j)}{\eta_{t}^{*}(k)} h^{\dagger}(s, d j, t, k),
$$

for $s \leqslant t \leqslant u$ in $[r, v], j, k, l \in \mathbb{R}^{d}$, where $h(t, k, u, d l)$ and $h^{\dagger}(s, d j, t, k)$ are the kernels associated to $\exp (-(u-t) H)$ and $\exp \left(-(t-s) H^{\dagger}\right)$. In the time homogeneous case (i.e. when $\eta, \eta^{*}$ depend trivially on time) this construction of Markov semi-groups in relation to time reversal goes back to [18] (see also [11] where it is applied to conditioned processes), but does not seem to have been the object of systematic studies when $\eta_{t}$ and $\eta_{t}^{*}$ are given as the solutions of "heat equations" for a general Lévy generator $H$ with potential. This also provides a construction of Bernstein processes [3] in the jump case, i.e. we construct $\mathbb{R}^{d}$-valued stochastic processes $\left(z_{t}\right)_{t \in[r, v]}$ that satisfy the relation

$$
P\left(z_{t} \in d k \mid \mathcal{P}_{s} \vee \mathcal{F}_{u}\right)=P\left(z_{t} \in d k \mid z_{s}, z_{u}\right), \quad r \leqslant s<t<u \leqslant v,
$$


where $\left(\mathcal{P}_{t}\right)_{t \in[r, v]}$, respectively $\left(\mathcal{F}_{t}\right)_{t \in[r, v]}$, denotes the increasing, respectively decreasing, filtration generated by

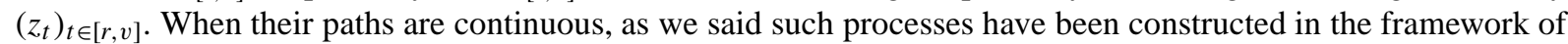
Euclidean quantum mechanics. We also show that for the class of potentials considered in this paper, the processes constructed are essentially the only Markovian Bernstein processes.

We proceed as follows. After recalling some notation on Lévy processes and their generators in Section 2, the main results of the paper are presented in Sections 3 and 4. The construction of Bernstein processes with jumps is given in Section 5. In Section 6, we compute the generators of Markovian bridges and derive the associated stochastic differential equations driven by Lévy processes. The uniqueness of Markovian Bernstein processes with jumps is discussed in Section 7. A variational characterization is obtained in Section 8. In particular, the construction provides time reversible jump diffusions whose law is given in terms of positive solutions of "heat equations" associated to the Schrödinger operator in the momentum representation.

\section{Notation - Lévy processes and generators}

We refer to the survey [21] and to the references therein for the notions recalled in this section. Let $V: \mathbb{R}^{d} \rightarrow \mathbb{C}$ such that $V(0) \geqslant 0$ and $\exp (-t V(q))$ is continuous in $\mathrm{q}$ and positive definite. The function $V$ admits the LévyKhintchine representation

$$
V(q)=a+i\langle c, q\rangle+\frac{1}{2}\langle q, q\rangle_{B}-\int_{\mathbb{R}^{d}}\left(e^{-i\langle q, y\rangle}-1+i\langle q, y\rangle 1_{\{|y| \leqslant 1\}}\right) \nu(d y),
$$

where $a, r \in \mathbb{R}^{d}, B$ is a positive definite $d \times d$ matrix, $\langle q, q\rangle_{B}=\langle B q, q\rangle$, and $v$ is a Lévy measure on $\mathbb{R}^{d} \backslash\{0\}$ satisfying $\int_{\mathbb{R}^{d}}\left(|y|^{2} \wedge 1\right) v(d y)<\infty$. In the following we assume without loss of generality that $a=0$, i.e. $V(0)=0$. Then the Lévy process is conservative, i.e. it has an infinite life time. Let $\xi_{t}$ denote the Lévy process with characteristic exponent $V(q)$, i.e. such that

$$
E\left[e^{-i\left\langle\xi_{t}, q\right\rangle}\right]=e^{-t V(q)}, \quad q \in \mathbb{R}^{d}, t \in \mathbb{R},
$$

or

$$
E\left[\exp \left(-i\left\langle\xi_{t}, q\right\rangle\right)\right]=\exp \left(-t\left(i\langle c, q\rangle+\frac{1}{2}\langle q, q\rangle_{B}-\int_{\mathbb{R}^{d}}\left(e^{-i\langle q, y\rangle}-1+i\langle q, y\rangle 1_{\{|y| \leqslant 1\}}\right) v(d y)\right)\right),
$$

$q \in \mathbb{R}^{d}, t \in \mathbb{R}$. The process $\left(\xi_{t}\right)_{t \in[r, v]}$ admits the (forward) Lévy-Itô decomposition with respect to the filtration $\left(\mathcal{P}_{t}\right)_{t \in[r, v]}$ :

$$
\xi_{t}=W_{t}+\int_{0}^{t} \int_{\{|y| \leqslant 1\}} y(\mu(d y, d s)-v(d y) d s)+\int_{0}^{t} \int_{\{|y| \geqslant 1\}} y \mu(d y, d s)+c t,
$$

where $W_{t}$ is a Brownian motion with covariance matrix $B$, and $\mu(d y, d s)$ is the Poisson random measure

$$
\mu(d y, d s)=\sum_{\Delta \xi_{s} \neq 0} \delta_{\left(\Delta \xi_{s}, s\right)}(d y, d s)
$$

with compensator $E[\mu(d y, d s)]=v(d y) d s$. Let $\mu_{t}$ denote the law of $\xi_{t}$, and let $\mu_{t}(d k)=\mu_{-t}(-d k)$ when $t<0$. The (forward) generator of $\left(\xi_{t}\right)_{t \in[r, v]}$ is the pseudo-differential operator

$$
-V(i \nabla) f(k)=\langle c, \nabla f(k)\rangle+\frac{1}{2} \Delta_{B} f(k)+\int_{\mathbb{R}^{d}}\left(f(k+y)-f(k)-\langle y, \nabla f(k)| 1_{\{|y| \leqslant 1\}}\right) v(d y),
$$


where $\Delta_{B}=\operatorname{div} B \nabla$. We shall also need the time reversed Lévy process $\left(\xi_{t}^{*}\right)_{t \in[0, v]}=\left(\xi_{v-t}\right)_{t \in[0, v]}$ whose (backward) generator is

$$
\begin{aligned}
-\bar{V}(\nabla) f(k) & =-V(-i \nabla) f(k) \\
& =-\langle c, \nabla f(k)\rangle+\frac{1}{2} \Delta_{B} f(k)+\int_{\mathbb{R}^{d}}\left(f(k-y)-f(k)+\langle y, \nabla f(k)| 1_{\{|y| \leqslant 1\}}\right) \nu(d y) .
\end{aligned}
$$

In view of applications to mathematical physics we consider a perturbation of the generator of $\left(\xi_{t}\right)_{t \in[r, v]}$ by a potential $U: \mathbb{R}^{d} \rightarrow \mathbb{R}$, continuous and bounded below:

Definition 2.1. Let $H=U+V(i \nabla)$, i.e. for $f \in \mathcal{S}\left(\mathbb{R}^{d}\right)$ :

$$
\begin{aligned}
H f(k)= & U(k) f(k)-\langle c, \nabla f(k)\rangle-\frac{1}{2} \Delta_{B} f(k) \\
& -\int_{\mathbb{R}^{d}}\left(f(k+y)-f(k)-\langle y, \nabla f(k)| 1_{\{|y| \leqslant 1\}}\right) \nu(d y), \quad k \in \mathbb{R}^{d} .
\end{aligned}
$$

The operator $V(i \nabla)$ is obtained from the potential $V$ by considering (Euclidean) momentum $\nabla$ as a variable. The potential $U$ is symmetrically deduced from a differential operator, e.g. the quadratic potential $U(k)=k^{2} / 2$ in momentum representation correspond to the Laplacian $\Delta$ in position representation. The adjoint $H^{\dagger}$ of $H$ with respect to the Lebesgue measure $d k$ is given by $H^{\dagger}=U+\bar{V}(\nabla)$ with $\bar{V}(q)=V(-q)$, i.e.

$$
\begin{aligned}
H^{\dagger} f(k)= & U(k) f(k)+\langle c, \nabla f(k)\rangle-\frac{1}{2} \Delta_{B} f(k) \\
& -\int_{\mathbb{R}^{d}}\left(f(k-y)-f(k)+\langle y, \nabla f(k)| 1_{\{|y| \leqslant 1\}}\right) v(d y), \quad k \in \mathbb{R}^{d} .
\end{aligned}
$$

If $c=0$, the operator $H$ is symmetric when $V$ is real-valued, that is when $v$ is symmetric with respect to $y \mapsto-y$, which is the case in theoretical physics.

Let $T_{t, u}, t<u$, respectively $T_{s, t}^{\dagger}, s<t$, denote the positive operator defined through the Feynman-Kac formula

$$
T_{t, u} f(k)=E\left[f\left(\xi_{u}\right) e^{-\int_{t}^{u} U\left(\xi_{\tau}\right) d \tau} \mid \xi_{t}=k\right]=E\left[f\left(k+\xi_{u-t}\right) e^{-\int_{0}^{u-t} U\left(k+\xi_{\tau}\right) d \tau}\right], \quad t<u,
$$

respectively

$$
T_{s, t}^{\dagger} f(k)=E\left[f\left(\xi_{s}^{*}\right) e^{-\int_{s}^{t} U\left(\xi_{\tau}^{*}\right) d \tau} \mid \xi_{t}^{*}=k\right]=E\left[f\left(k-\xi_{t-s}\right) e^{-\int_{0}^{t-s} U\left(k-\xi_{t-s-\tau}\right) d \tau}\right], \quad s<t .
$$

Since $-V(i \nabla)$ is the (forward) generator of $\left(\xi_{t}\right)_{t \in[0, u]}$ and $-\bar{V}(\nabla)=-V(-i \nabla)$ is the (backward) generator of the time reversed Lévy process $\left(\xi_{s}^{*}\right)_{s \in[0, t]}=\left(\xi_{t-s}\right)_{s \in[0, t]}$, we have

$$
\frac{\partial}{\partial u} T_{t, u}=-T_{t, u} H, \quad \text { and } \quad \frac{\partial}{\partial s} T_{s, t}^{\dagger}=T_{s, t}^{\dagger} H^{\dagger},
$$

or $T_{t, u}=\exp (-(u-t) H)$ and $T_{s, t}^{\dagger}=\exp \left(-(t-s) H^{\dagger}\right)$, i.e. these semi-groups are time homogeneous since $V$ and $U$ are independent of time.

We denote by $h^{\dagger}(s, d j, t, k)$ and $h(t, k, u, d l), 0<s<t<u, j, k, l \in \mathbb{R}^{d}$, the "integral kernels" of $\exp \left(-(t-s) H^{\dagger}\right)$ and $\exp (-(u-t) H)$, defined by

$$
\exp \left(-(t-s) H^{\dagger}\right) f(k)=\int_{\mathbb{R}^{d}} f(j) h^{\dagger}(s, d j, t, k), \quad \text { and } \quad \exp (-(u-t) H) f(k)=\int_{\mathbb{R}^{d}} f(l) h(t, k, u, d l) .
$$




\section{Examples.}

(1) Deterministic process.

Here, $U$ does not necessarily vanish. Then $B=0$ and $v=0$ but $c \neq 0$. So $V(q)=i c q$ and

$$
H f(k)=-c \nabla f(k), \quad H^{\dagger} f(k)=c \nabla f(k),
$$

with integral kernels

$$
\begin{aligned}
& h^{\dagger}(s, d j, t, k)=\delta_{k-c(t-s)}(d j) e^{-\int_{s}^{t} U(k+c(\tau-t)) d \tau}, \\
& h(t, k, u, d l)=\delta_{k+c(u-t)}(d l) e^{-\int_{t}^{u} U(k+c(\tau-t)) d \tau} .
\end{aligned}
$$

(2) Lévy processes $(U=0)$.

Without hypothesis on the absolute continuity of $\mu_{t}, e^{-(u-t) H}$ and $e^{-(t-s) H^{\dagger}}$ are respectively given by convolution with the law $\mu_{t}$ of the Lévy process:

$$
\int_{\mathbb{R}^{d}} \eta_{s}^{*}(d j) h^{\dagger}(s, j, t, d k)=\eta_{s}^{*} * \mu_{t-s}(d k), \quad s<t,
$$

and

$$
\int_{\mathbb{R}^{d}} \eta_{u}(d l) h(t, d k, u, l)=\eta_{u} * \mu_{t-u}(d k), \quad t<u .
$$

If moreover $\eta_{s}^{*}(d j)=\eta_{s}^{*}(j) \lambda(d j), \eta_{u}(d l)=\eta_{u}(l) \lambda(d l)$ and $\mu_{t}(d k)=\mu_{t}(k) \lambda(d k)$ are absolutely continuous with respect to $\lambda$, then

$$
h(s, j, t, d k)=\mu_{t-s}(k-j) \lambda(d k),
$$

and

$$
h(t, d k, u, l)=\mu_{u-t}(l-k) \lambda(d k) .
$$

(3) General case $(U \neq 0)$.

We have, by definition

$$
\begin{aligned}
\int_{\mathbb{R}^{d}} h(t, k, u, d l) f(l) & =e^{-(u-t) H} f(k) \\
& =E\left[f\left(\xi_{u}\right) e^{-\int_{t}^{u} U\left(\xi_{\tau}\right) d \tau} \mid \xi_{t}=k\right]=E\left[f\left(\xi_{u-t}+k\right) e^{-\int_{0}^{u-t} U\left(k+\xi_{\tau}\right) d \tau}\right] \\
& =\int_{\mathbb{R}^{d}} E\left[f\left(\xi_{u-t}+k\right) e^{-\int_{0}^{u-t} U\left(k+\xi_{\tau}\right) d \tau} \mid \xi_{u-t}=l\right] \mu_{u-t}(d l) \\
& =\int_{\mathbb{R}^{d}} f(k+l) E\left[e^{-\int_{0}^{u-t} U\left(k+\xi_{\tau}\right) d \tau} \mid \xi_{u-t}=l\right] \mu_{u-t}(d l) \\
& =\int_{\mathbb{R}^{d}} f(l) E\left[e^{-\int_{0}^{u-t} U\left(k+\xi_{\tau}\right) d \tau} \mid \xi_{u-t}=l-k\right] \mu_{u-t}(-k+d l),
\end{aligned}
$$

where $\mu_{u-t}(-k+d l)$ denotes the image measure of $\mu_{u-t}$ under $l \mapsto k+l$ (i.e. $\mu_{u-t}(-k+d l)=\mu_{u-t}(l-$ $k) \lambda(d l)$ if $\mu_{u-t}(d l)$ has the density $\mu_{u-t}(l)$ with respect to $\left.\lambda\right)$. Consequently we obtain

$$
h(t, k, u, d l)=\alpha(u-t, k, l) \mu_{u-t}(-k+d l),
$$


with

$$
\alpha(u-t, k, l)=E\left[e^{-\int_{0}^{u-t} U\left(k+\xi_{\tau}\right) d \tau} \mid \xi_{u-t}=l-k\right] .
$$

Similarly we have

$$
h^{\dagger}(s, d j, t, k)=\alpha(t-s, j, k) \mu_{t-s}(-k-d j) .
$$

We end this section with a lemma that will be useful to determine the forward (respectively backward) drift of a backward (respectively forward) Lévy process in Section 3.2.

Lemma 2.2. Assume that $\mu_{t}(d k)=\mu_{t}(k) \lambda(d k)$ is absolutely continuous with respect to a reference measure $\lambda$ which is invariant by translations. We have

$$
\int_{\mathbb{R}^{d}} y \frac{\mu_{t}(k-y)}{\mu_{t}(k)} v(d y)=\frac{k}{t}-c+B \nabla \log \mu_{t}(k)+\int_{\mathbb{R}^{d}} y 1_{\{|y| \leqslant 1\}} v(d y), \quad \lambda(d k) \text {-a.e. },
$$

and

$$
\int_{\mathbb{R}^{d}} y \frac{\mu_{t-v}(k+y)}{\mu_{t-v}(k)} v(d y)=-\frac{k}{v-t}-c-B \nabla \log \mu_{t-v}(k)+\int_{\mathbb{R}^{d}} y 1_{\{|y| \leqslant 1\}} v(d y), \quad \lambda(d k) \text {-a.e. }
$$

Proof. We have for all $q \in \mathbb{R}^{d}$ :

$$
\begin{aligned}
-i \int_{\mathbb{R}^{d}} k e^{-i k q} \mu_{t}(k) d k= & \nabla_{q} e^{-t V(q)}=-t e^{-t V(q)} \nabla V(q) \\
= & -t\left(i c+B q+i \int_{\mathbb{R}^{d}} y\left(e^{-i q y}-1_{\{|y| \leqslant 1\}}\right) v(d y)\right) \int_{\mathbb{R}^{d}} e^{-i k q} \mu_{t}(k) d k \\
= & -i t \int_{\mathbb{R}^{d}} \int_{\mathbb{R}^{d}} y e^{-i k q} \mu_{t}(k-y) d k v(d y) \\
& -t(i c+B q) \int_{\mathbb{R}^{d}} e^{-i k q} \mu_{t}(k) d k+i t \int_{\mathbb{R}^{d}} e^{-i k q} \mu_{t}(k) \int_{\mathbb{R}^{d}} y 1_{\{|y| \leqslant 1\}} v(d y) d k \\
= & -i t \int_{\mathbb{R}^{d}} e^{-i k q}\left(\int_{\mathbb{R}^{d}} y \mu_{t}(k-y) d k v(d y)+c \mu_{t}(k)\right. \\
& \left.-B \nabla \mu_{t}(k)-\mu_{t}(k) \int_{\mathbb{R}^{d}} y 1_{\{|y| \leqslant 1\}} v(d y)\right) .
\end{aligned}
$$

The second relation is proved from $\mu_{t-v}(k)=\mu_{v-t}(-k)$ and

$\left[\nabla \log \mu_{v-t}\right](-k)=-\nabla \log \mu_{v-t}(-k)=-B \nabla \log \mu_{t-v}(k)$. 


\section{Construction of Markovian bridges - main results}

One of the objectives of this paper is the proof of Proposition 3.1 below. Assume that

- $h^{\dagger}(s, d j, t, k)=h^{\dagger}(s, j, t, k) \lambda(d j)$ and $h(t, k, u, d l)=h(t, k, u, l) \lambda(d l)$ are absolutely continuous with respect to $\lambda$,

- $H$ and $H^{\dagger}$ are mutually adjoint under $\lambda$, i.e. $h^{\dagger}(s, j, t, k)=h(s, j, t, k)$,

$-h^{\dagger}(s, j, t, k)=h(s, j, t, k)$ is continuous in $(j, k)$ and strictly positive for all $0<s<t$.

Let $z_{t^{-}}$denotes the left limit of $z$ at $t \in[r, v]$. The following proposition holds under the assumptions (A) and (B) of Section 3.2, before Proposition 3.5.

Proposition 3.1. Let $\pi_{r}(d k)$ and $\pi_{v}(d k)$ be two given probability measures on $\mathbb{R}^{d}$, which are assumed to be absolutely continuous with a.e. strictly positive densities with respect to a fixed reference measure $\lambda$. There exists $a \mathbb{R}^{d}$-valued process $\left(z_{t}\right)_{t \in[r, v]}$ with initial distribution $\pi_{r}(d k)$ and final distribution $\pi_{v}(d k)$, driven by $\left(\xi_{t}\right)_{t \in[r, v]}$, i.e. such that $\left(z_{t}\right)_{t \in[r, v]}$ solves in the weak sense the stochastic integro-differential equation

$$
\begin{aligned}
d z_{t}= & c d t+d W_{t}+\int_{\mathbb{R}^{d}} y\left(\mu(d y, d t)-\frac{\eta_{t}\left(z_{t^{-}}+y\right)}{\eta_{t}\left(z_{t^{-}}\right)} 1_{\{|y| \leqslant 1\}} v(d y) d t\right) \\
& +\int_{\mathbb{R}^{d}} y\left(\frac{\eta_{t}\left(z_{t^{-}}+y\right)-\eta_{t}\left(z_{t^{-}}\right)}{\eta_{t}\left(z_{t^{-}}\right)}\right) 1_{\{|y| \leqslant 1\}} v(d y) d t+B \nabla \log \eta_{t}\left(z_{t^{-}}\right) d t,
\end{aligned}
$$

and the law of $z_{t}$ at time $t$ is $\eta_{t}(k) \eta_{t}^{*}(k) \lambda(d k)$, where

$$
\eta_{t}=e^{-(v-t) H} \eta_{v}, \quad \eta_{t}^{*}=e^{-(t-r) H^{\dagger}} \eta_{r}^{*}, \quad r \leqslant t \leqslant v,
$$

$W$ is a Brownian motion with covariance $B$, the canonical point process $\mu(d y, d t)$ has compensator $\frac{\eta_{t}\left(z_{t}-y\right)}{\eta_{t}\left(z_{t^{-}}\right)} \nu(d y) d t$, and $\eta_{r}^{*}, \eta_{v}$ are two positive initial and final conditions determined from $\pi_{r}$ and $\pi_{v}$.

Moreover the process $\left(z_{t}\right)_{t \in[r, v]}$ in question is a Bernstein process, i.e.

$$
P\left(z_{t} \in d k \mid \mathcal{P}_{s} \vee \mathcal{F}_{u}\right)=P\left(z_{t} \in d k \mid z_{s}, z_{u}\right), \quad s<t<u,
$$

and the joint law $P\left(z_{r} \in A, z_{v} \in B\right)$, for $A, B$ two Borelians of $\mathbb{R}^{d}$, is of the form

$$
P\left(z_{r} \in A, z_{v} \in B\right)=\int_{A \times B} \eta_{r}^{*}(i) h(r, i, v, m) \eta_{v}(m) \lambda(d i) \lambda(d m) .
$$

Note that we have $H^{\dagger} f(k)=H \tilde{f}(-k)$, where $\tilde{f}(k)=f(-k), k \in \mathbb{R}^{d}$. As a consequence, $\eta_{t}^{*}(k)=\tilde{\eta}_{v-(t-r)}(-k)$, where $\left(\tilde{\eta}_{t}\right)_{t \in[r, v]}$ is solution of

$$
\left\{\begin{array}{l}
\frac{\partial}{\partial t} \tilde{\eta}_{t}=H \tilde{\eta}_{t} \\
\tilde{\eta}_{v}(k)=\eta_{r}^{*}(-k)
\end{array}\right.
$$

and $\eta_{t}(k)=\tilde{\eta}_{v-(t-r)}^{*}(-k)$, where $\left(\tilde{\eta}_{t}^{*}\right)_{t \in[r, v]}$ is solution of

$$
\left\{\begin{array}{l}
\frac{\partial}{\partial t} \tilde{\eta}_{t}^{*}=-H^{\dagger} \tilde{\eta}_{t}^{*} \\
\tilde{\eta}_{r}^{*}(k)=\eta_{v}(-k)
\end{array}\right.
$$

Hence $\left(z_{t}\right)_{t \in[r, v]}$ has same law as the reversed process $\left(-\tilde{z}_{v-(t-r)}\right)_{t \in[r, v]}$, where $\left(\tilde{z}_{t}\right)_{t \in[r, v]}$ is solution of the same problem, with final law $\pi_{r}(-d k)$ at time $v$ and initial law $\pi_{v}(-d k)$ at time $r$. If $v$ is symmetric then $H=H^{\dagger}$, and 
if moreover $\eta_{r}^{*}, \eta_{v}$ are symmetric then $\pi_{r}, \pi_{v}$ are symmetric and $\eta_{t}^{*}(k)=\tilde{\eta}_{v-(t-r)}(k), \eta_{t}(k)=\tilde{\eta}_{v-(t-r)}^{*}(k)$. In this case, $\left(z_{t}\right)_{t \in[r, v]}$ is reversible, i.e. it has same law as $\left(\tilde{z}_{v-(t-r)}\right)_{t \in[r, v]}$.

We will also prove a uniqueness result, i.e. if $\left(z_{t}\right)_{t \in[r, v]}$ is a Markovian Bernstein process with Bernstein kernel $h(s, j, t, d k, u, l)=P\left(z_{t} \in d k \mid z_{s}=j, z_{u}=l\right)$ such that

$$
h(s, j, t, d k, u, l) h(s, j, u, d l)=h(s, j, t, d k) h(t, k, u, d l),
$$

or

$$
h(s, j, t, d k, u, l) h^{\dagger}(s, d j, u, l)=h^{\dagger}(s, d j, t, k) h^{\dagger}(t, d k, u, l),
$$

$s \leqslant t \leqslant u, j, k, l \in \mathbb{R}^{d}$, then there exists positive density functions $\eta_{r}^{*}(i)$ and $\eta_{v}(m)$ such that

$$
P\left(z_{r} \in A, z_{v} \in B\right)=\int_{A \times B} \eta_{r}^{*}(i) h(r, i, v, m) \eta_{v}(m) \lambda(d i) \lambda(d m),
$$

cf. Theorem 7.1. These results will be precisely stated in different forms and under weaker assumptions in the following section. Proofs will be provided afterwards in several steps, which consist of more refined statements.

\subsection{Existence of Markovian bridges}

In the following result, $h(t, k, u, d l)$ and $h^{\dagger}(s, d j, t, k)$ need not be absolutely continuous with respect to a fixed reference measure $\lambda$.

Theorem 3.2. Let $\lambda$ be a fixed reference measure such that $H$ and $H^{\dagger}$ are adjoint with respect to $\lambda$, and let $\eta_{r}^{*}, \eta_{v}: \mathbb{R}^{d} \rightarrow \mathbb{R}_{+}$be two $\lambda$-a.e. strictly positive initial and final conditions such that for some $t \in[r, v]$ (and therefore for any such $t$ ),

$$
\int_{\mathbb{R}^{d}} \eta_{t}^{*}(k) \eta_{t}(k) \lambda(d k)=1,
$$

where

$$
\eta_{t}^{*}(k)=e^{-(t-r) H^{\dagger}} \eta_{r}^{*}(k)=\int_{\mathbb{R}^{d}} \eta_{r}^{*}(i) h^{\dagger}(r, d i, t, k),
$$

and

$$
\eta_{t}(k)=e^{-(v-t) H} \eta_{v}(k)=\int_{\mathbb{R}^{d}} \eta_{v}(m) h(t, k, v, d m), \quad r \leqslant t \leqslant v .
$$

Then there exists a $\mathbb{R}^{d}$-valued process $\left(z_{t}\right)_{t \in[r, v]}$ whose density at time $t$ with respect to $\lambda$ is $\rho_{t}(k)=\eta_{t}^{*}(k) \eta_{t}(k)$, which is forward and backward Markovian, with forward transition kernel

$$
p(t, k, u, d l)=\frac{\eta_{u}(l)}{\eta_{t}(k)} h(t, k, u, d l),
$$

and backward transition kernel

$$
p^{*}(s, d j, t, k)=\frac{\eta_{s}^{*}(j)}{\eta_{t}^{*}(k)} h^{\dagger}(s, d j, t, k) .
$$

In particular, the initial and final laws of $\left(z_{t}\right)_{t \in[r, v]}$ are $\pi_{r}(d i)=\eta_{r}(i) \eta_{r}^{*}(i) \lambda(d i)$ and $\pi_{v}(d m)=\eta_{v}(m) \eta_{v}^{*}(m) \lambda(d m)$. 
The above functions $\eta_{t}^{*}(k)$ and $\eta_{t}(k)$ satisfy the partial integro-differential equations

$$
-\frac{\partial \eta_{t}^{*}}{\partial t}(k)=H^{\dagger} \eta_{t}^{*}(k) \quad \text { and } \quad \frac{\partial \eta_{t}}{\partial t}(k)=H \eta_{t}(k), \quad t \in[r, v] .
$$

The proof of Theorem 3.2 follows from Propositions 5.1 and 5.2 below. Once Theorem 3.2 is proved, Proposition 3.1 follows from 3.5 and Theorem 3.3 below which states that given two probability measures $\pi_{r}(d i)=\pi_{r}(i) \lambda(d i)$ and $\pi_{v}(d m)=\pi_{v}(m) \lambda(d m)$, absolutely continuous with respect to $\lambda$, it is possible to determine two positive initial and final functions $\eta_{r}^{*}, \eta_{v}: \mathbb{R}^{d} \rightarrow \mathbb{R}_{+}$from the data of the initial and final laws $\pi_{r}, \pi_{v}$ of the process, such that

$$
\pi_{r}(i)=\eta_{r}^{*}(i) \eta_{r}(i), \quad \pi_{v}(m)=\eta_{v}(m) \eta_{v}^{*}(m),
$$

where

$$
\eta_{r}(i)=\int_{\mathbb{R}^{d}} \eta_{v}(m) h(r, i, v, d m),
$$

and

$$
\eta_{v}^{*}(m)=\int_{\mathbb{R}^{d}} \eta_{r}^{*}(i) h^{\dagger}(r, d i, v, m),
$$

provided $h(s, k, t, d j)$ and $h^{\dagger}(s, d j, t, k)$ are absolutely continuous with respect to $\lambda$ :

$$
\begin{aligned}
& h(s, k, t, d j)=h(s, k, t, j) \lambda(d j), \\
& h^{\dagger}(s, d j, t, k)=h^{\dagger}(s, j, t, k) \lambda(d j),
\end{aligned}
$$

with $h(s, k, t, j)=h^{\dagger}(s, k, t, j)$ since $H$ is adjoint of $H^{\dagger}$ with respect to $\lambda$. More precisely we have the following result, cf. Theorem 1 of [5], Theorem 3.2 of [24], and Theorem 3.4 of [31]:

Theorem 3.3. Let $\pi_{r}$ and $\pi_{v}$ be two probability measures. Assume that $h(s, j, k, t)$ is a continuous in $(j, k)$ and strictly positive function. Then there exist two measures $\eta_{r}^{*}(d i)$ and $\eta_{v}(d m)$ such that

$$
\pi_{r}(d i)=\eta_{r}^{*}(d i) \int_{\mathbb{R}^{d}} h(r, i, v, m) \eta_{v}(d m),
$$

and

$$
\pi_{v}(d m)=\eta_{v}(d m) \int_{\mathbb{R}^{d}} h(r, i, v, m) \eta_{r}^{*}(d i) .
$$

We present several families of processes satisfying the above hypothesis, starting with the simplest examples. Note that in the first example, the mutual adjointness of $H$ and $H^{\dagger}$ with respect to the (Lebesgue) measure $\lambda$ is satisfied without requiring the absolute continuity of $h(t, k, u, d l)$ and $h^{\dagger}(s, d j, t, k)$ with respect to $\lambda$. We will also present some examples where the initial and final laws can not be arbitrarily chosen, when the hypothesis of Theorem 3.3 are not fulfilled. This list of examples includes the classical Brownian bridge, however the aim of this paper is not to focus on the Brownian case which has already been the object of several studies, cf. [6,7,24,2,31,9].

\section{Examples.}

(1) Deterministic process. 
The adjoint relation between $H$ and $H^{\dagger}$ is satisfied in the deterministic case for $\lambda$ the Lebesgue measure, i.e.

$$
\begin{array}{ll}
h^{\dagger}(s, d j, t, k)=e^{-\int_{s}^{t} U(k+c(\tau-t)) d \tau} \delta_{k-c(t-s)}(d j), & r \leqslant s<t \leqslant v, \\
h(t, k, u, d l)=e^{-\int_{t}^{u} U(k+c(\tau-t)) d \tau} \delta_{k+c(u-t)}(d l), & r \leqslant t<u \leqslant v .
\end{array}
$$

Therefore, for any $r<s<t<u<v$,

$$
\begin{aligned}
& \eta_{t}^{*}(k)=\eta_{s}^{*}(k-c(t-s)) e^{-\int_{s}^{t} U(k+c(\tau-t)) d \tau}, \\
& \eta_{t}(k)=\eta_{u}(k+c(u-t)) e^{-\int_{t}^{u} U(k+c(\tau-t)) d \tau .}
\end{aligned}
$$

Applying (3.7) and (3.8) successively in $t=s$ and $t=u$ we obtain several expressions for the density of $z_{t}$ at time $t$ with respect to the Lebesgue measure $\lambda$ :

$$
\begin{aligned}
\eta_{t}^{*}(k) \eta_{t}(k) & =\eta_{s}^{*}(k-c(t-s)) \eta_{u}(k-c(t-u)) e^{-\int_{s}^{u} U(k-c(t-\tau)) d \tau} \\
& =\eta_{s}^{*}(k-c(t-s)) \eta_{s}(k-c(t-s)) \\
& =\eta_{u}^{*}(k-c(t-u)) \eta_{u}(k-c(t-u)),
\end{aligned}
$$

Note that here $h(s, k, t, d l)$ is not absolutely continuous with respect to the Lebesgue measure $\lambda(d l)$ and that it is clearly not possible to choose independently the initial and final laws.

(2) Lévy bridges from $a \in \mathbb{R}^{d}$ to $b \in \mathbb{R}^{d}$.

Take $U=0$, and assume that $\mu_{t}(d k), t>0$, has a density with respect to a fixed reference measure $\lambda$, i.e. $\mu_{t}(d k)=\mu_{t}(k) \lambda(d k)$. Then taking

$$
\eta_{t}^{*}(k)=C(r, v, a, b) \mu_{t-r}(k-a), \quad \eta_{t}(k)=\mu_{t-v}(k-b)=\mu_{v-t}(b-k),
$$

where $C(r, v, a, b)$ is a normalization constant, the resulting density at time $t$ with respect to $\lambda$ is

$$
\eta_{t}^{*}(k) \eta_{t}(k)=\frac{\mu_{t-r}(k-a) \mu_{v-t}(b-k)}{\mu_{v-r}(b-a)},
$$

where $C(r, v, a, b)=1 / \mu_{v-r}(b-a)$. In this way we recover the density of the Lévy bridge in the usual sense, obtained by conditioning the Lévy process $\left(\xi_{t}\right)_{t \in[r, v]}$ by $\xi_{r}=a$ and $\xi_{v}=b$ :

$$
\begin{aligned}
d P\left(\xi_{t}=k \mid \xi_{r}=a, \xi_{v}=b\right) & =\frac{d P\left(\xi_{t} \in d k, \xi_{r} \in d a, \xi_{v} \in d b\right)}{P\left(\xi_{r} \in d a, \xi_{v} \in d b\right)} \\
& =\frac{\mu_{t-r}(k-a) \mu_{v-t}(b-k)}{\mu_{v-r}(b-a)} \lambda(d k) .
\end{aligned}
$$

This example includes the Poisson and Brownian bridges below. Note that the absolute continuity of $\eta_{t}(d k)$ with respect to $\lambda$ at the initial and final times is not always satisfied since $\eta_{r} \eta_{r}^{*}(d k)=\delta_{a}(d k), \eta_{v} \eta_{v}^{*}(d k)=$ $\delta_{b}(d k)$, for example it is satisfied for the Poisson bridge but not for the Brownian bridge.

(3) Poisson bridge starting from $a \in \mathbb{N}$ at time $r$ and ending at $b \in \mathbb{N}$ at time $v$.

Here the kinetic term $U$ vanishes, as well as $B$, and $c=1$. Moreover $v=\delta_{1}$, so $V(q)=-\left(e^{-i q}-1\right)$ and we have

$$
-H f(k)=f(k+1)-f(k), \quad-H^{\dagger} f(k)=f(k-1)-f(k) .
$$

The standard Poisson bridge provides another example where the initial and final laws cannot be chosen arbitrarily, this time because $h(t, k, u, l)$ is not everywhere strictly positive. Take $U=0, c=1, v=\delta_{1}$, and the reference measure

$$
\lambda=\sum_{n=-\infty}^{+\infty} \delta_{n} .
$$


The simple Poisson bridge with $z_{r}=a$ and $z_{v}=b$ is constructed from the boundary conditions

$$
\eta_{r}^{*}=C(r, v, a, b) 1_{\{a\}}, \quad \eta_{v}=1_{\{b\}},
$$

where $C(r, v, a, b)$ is a normalization constant. Then

$$
\begin{aligned}
& \eta_{t}^{*}(k)=C(r, v, a, b) \mu_{t-r}(k-a)=C(r, v, a, b) e^{-(t-r)} \frac{(t-r)^{k-a}}{(k-a) !} 1_{\{k \geqslant a\}}, \\
& \eta_{t}(k)=\mu_{v-t}(b-k)=e^{-(v-t)} \frac{(v-t)^{b-k}}{(b-k) !} 1_{[0, b]}(k),
\end{aligned}
$$

with the convention $0^{0}=1$. The resulting density at time $t$ with respect to $\lambda$ is, therefore,

$$
\eta_{t}^{*}(k) \eta_{t}(k)=1_{[a, b]}(k)\left(\begin{array}{l}
b-a \\
k-a
\end{array}\right)\left(\frac{t-r}{v-r}\right)^{k-a}\left(\frac{v-t}{v-r}\right)^{b-k}, \quad r \leqslant t \leqslant v,
$$

which is the expected binomial law on $\{a, \ldots, b\}$ with parameter $(t-r) /(v-r)$, obtained by conditioning, with $C(r, v, a, b)=e^{v-r} \frac{(b-a) !}{(v-r)^{(b-a)}}$. Note that here, $h(t, k, u, l)=e^{-(u-t)} \frac{(u-t)^{l-k}}{(l-k) !} 1_{[0, l]}(k)$ is not $(\lambda \otimes \lambda)(d l, d k)$ strictly positive, and the initial and final laws cannot be chosen arbitrarily, e.g. one cannot have $b<a$. Also this setting is not directly relevant to physics in the momentum representation since $U=0$.

(4) Gamma bridge starting from $a \in \mathbb{R}_{+}$at time $r$ and ending at $b \in \mathbb{R}_{+}, a<b$, at time $v$. We have $V(q)=$ $\log (1+i q / \beta)$ where $\beta$ is a strictly positive parameter, and $v(d y)=y^{-1} e^{-\beta y} 1_{[0, \infty)}(y) d y$, with $B=0, a=0$, $c=\int_{0}^{1} e^{-\beta y} y^{-1} d y$. Moreover $\mu_{t}(k)=1_{[0, \infty)}(k) \frac{\beta^{t}}{\Gamma(t)} k^{t-1} e^{-\beta k}$, hence

$$
\eta_{t}^{*}(k) \eta_{t}(k)=\frac{1}{b-a} \frac{\Gamma(v-r)}{\Gamma(t-r) \Gamma(v-t)}\left(\frac{k-a}{b-a}\right)^{t-r-1}\left(\frac{b-k}{b-a}\right)^{v-t-1},
$$

which is the density at time $t$ of the Gamma bridge $\left(a+(b-a) \xi_{t-r} / \xi_{v-r}\right)_{t \in[r, v]}$, in other terms $\left(z_{t}-a\right) /(b-a)$ has a beta distribution with parameters $(t-r, v-t), t \in[r, v]$.

(5) Brownian bridge.

The Brownian bridge starting at $a \in \mathbb{R}$ and ending at $b \in \mathbb{R}$ is constructed by taking $U=0, \mu_{t}(k)=$ $\frac{1}{\sqrt{2 \pi(t-r)}} e^{-\frac{1}{2} k^{2} /(t-r)}$, and

$$
\eta_{t}^{*}(k)=\frac{C(r, v, a, b)}{\sqrt{2 \pi(t-r)}} e^{-\frac{1}{2}(k-a)^{2} /(t-r)}, \quad \eta_{t}(k)=\frac{1}{\sqrt{2 \pi(v-t)}} e^{-\frac{1}{2}(b-k)^{2} /(v-t)},
$$

with

$$
C(r, v, a, b)=\sqrt{2 \pi(v-r)} e^{\frac{1}{2}(b-a)^{2} /(v-r)} .
$$

The product $\eta_{t}(k) \eta_{t}^{*}(k)$ gives the usual density of the Brownian bridge with respect to the Lebesgue measure:

$$
\eta_{t}(k) \eta_{t}^{*}(k)=\frac{\sqrt{v-r}}{\sqrt{2 \pi(t-r)(v-t)}} \exp \left(-\frac{v-r}{2(t-r)(v-t)}\left(k-\frac{a(v-t)+b(t-r)}{v-r}\right)^{2}\right) .
$$

(6) Forward and backward Lévy processes $(U=0)$.

This example includes the forward and backward Wiener and Poisson processes as particular cases. Taking $\eta_{r}^{*}(d i)=\mu_{r}(d i)$ and $\eta_{v}(m)=1$, we have

$$
\eta_{t}^{*}(d k)=\eta_{r}^{*} * \mu_{t-r}(d k)=\mu_{t}(d k), \quad \eta_{t}(k)=1, \quad r<t<v,
$$

hence $\left(z_{t}\right)_{t \in[r, v]}$ is the (forward) Lévy process $\left(\xi_{t}\right)_{t \in[r, v]}: z_{t}=\xi_{t}, r<t<v$.

If $\eta_{v}(d m)=\mu_{0}(d m)$ and $\eta_{r}^{*}(i)=1$, we have

$$
\eta_{t}(d k)=\eta_{v} * \mu_{t-v}(d k)=\mu_{t-v}(d k), \quad \eta_{t}^{*}(k)=1, \quad r<t<v,
$$


hence $\left(z_{t}\right)_{t \in[r, v]}$ is a backward Lévy process. This is an example of process with initial law $\mu_{r-v}(d k)$ and final condition $z_{v}=0$, respectively initial law $\mu_{r}(d k)$ and final law $\mu_{v}(d k)$.

(7) Processes with densities with respect to the Lebesgue measure.

Here, $U$ does not necessarily vanish. From (2.3) and (2.4), the absolute continuity conditions (3.5) and (3.6) are satisfied if the law of $\xi_{t}, t>0$, has a density with respect to the Lebesgue measure, e.g. in the case of stable processes (namely such that $V(q)=c|q|^{\alpha}$ for some $\alpha \in(0,2]$ and $c>0$ ), and for Lévy processes with Brownian component $(B \neq 0)$. Moreover $H$ is adjoint of $H^{\dagger}$ with respect to $\lambda$ when $\lambda$ is the Lebesgue measure.

(8) General case $(U \neq 0)$.

The condition $U \neq 0$ is necessary in the context of Euclidean quantum mechanics. If $\lambda$ is a given measure (not necessarily the Lebesgue measure), we may work under the absolute continuity hypothesis

$$
\begin{aligned}
& h(t, k, u, d l)=h(t, k, u, l) \lambda(d l), \quad \lambda(d k) \text {-a.e., } \\
& h^{\dagger}(s, d j, t, k)=h^{\dagger}(s, j, t, k) \lambda(d j), \quad \lambda(d k) \text {-a.e. }
\end{aligned}
$$

which imply that $H$ and $H^{\dagger}$ are also adjoint with respect to $\lambda$ if $h(s, j, t, k)=h^{\dagger}(t, k, s, j)$ :

$$
h(s, j, t, d k) \lambda(d j)=h(s, j, t, k) \lambda(d k) \lambda(d j)=h^{\dagger}(s, j, t, k) \lambda(d k) \lambda(d j)=h^{\dagger}(s, d j, t, k) \lambda(d k) .
$$

In view of (2.3) and (2.4), conditions (3.9) and (3.10) are satisfied in particular if $\mu_{t-s}(-j+d k$ ) has a density with respect to $\lambda(d k), \lambda(d j)$-a.e. This condition will be satisfied e.g. if $\lambda$ is absolutely continuous under the translation $k \mapsto j+k, \lambda(d j)$-a.e., and $\mu_{t-s}$ is absolutely continuous with respect to $\lambda$ :

$$
\mu_{t-s}(d k)=\mu_{t-s}(k) \lambda(d k) \text {. }
$$

This hypothesis is satisfied, in particular, for the Poisson bridge, cf. Example 3 above, with $\mu_{t-s}(k)=$

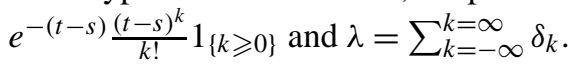

\subsection{Stochastic differential equations and generators}

In this section we present the description of Markovian bridges of Theorem 3.2 in terms of forward and backward stochastic integro-differential equations driven by $\left(\xi_{t}\right)_{t \in[r, v]}$. Let for $f \in \mathcal{S}\left(\mathbb{R}^{d}\right)$ and $g: \mathbb{R}^{d} \mapsto(0, \infty)$ :

$$
\begin{aligned}
\mathcal{L}_{g} f(k)= & \langle c, \nabla f(k)\rangle+\frac{1}{2} \Delta_{B} f(k)+\int_{\mathbb{R}^{d}}\left(f(k+y)-f(k)-\langle y, \nabla f(k)| 1_{\{|y| \leqslant 1\}} \frac{g(k+y)}{g(k)} v(d y)\right. \\
& +\int_{\mathbb{R}^{d}} \frac{g(k+y)-g(k)}{g(k)}\langle y, \nabla f(k)| 1_{\{|y| \leqslant 1\}} v(d y)+\langle\nabla \log g(k), \nabla f(k)\rangle_{B},
\end{aligned}
$$

and

$$
\begin{aligned}
\mathcal{L}_{g}^{*} f(k)= & \langle c, \nabla f(k)\rangle-\frac{1}{2} \Delta_{B} f(k)-\int_{\mathbb{R}^{d}}\left(f(k-y)-f(k)+\langle y, \nabla f(k)| 1_{\{|y| \leqslant 1\}}\right) \frac{g(k-y)}{g(k)} v(d y) \\
& +\int_{\mathbb{R}^{d}} \frac{g(k-y)-g(k)}{g(k)}\langle y, \nabla f(k)| 1_{\{|y| \leqslant 1\}} v(d y)-\langle\nabla \log g(k), \nabla f(k)\rangle_{B} .
\end{aligned}
$$

The following result is a consequence of Proposition 6.2, which will be proved in Section 6.

Proposition 3.4. The process $\left(z_{t}\right)_{t \in[r, v]}$ constructed in Theorem 3.2 admits the forward infinitesimal generator, for $f \in \mathcal{S}\left(\mathbb{R}^{d}\right)$ : 


$$
\begin{aligned}
\mathcal{L}_{\eta_{t}} f(k)= & \langle c, \nabla f(k)\rangle+\frac{1}{2} \Delta_{B} f(k)+\int_{\mathbb{R}^{d}}\left(f(k+y)-f(k)-\langle y, \nabla f(k)| 1_{\{|y| \leqslant 1\}}\right) \frac{\eta_{t}(k+y)}{\eta_{t}(k)} v(d y) \\
& +\int_{\mathbb{R}^{d}} \frac{\eta_{t}(k+y)-\eta_{t}(k)}{\eta_{t}(k)}\langle y, \nabla f(k)| 1_{\{|y| \leqslant 1\}} v(d y)+\left\langle\nabla \log \eta_{t}(k), \nabla f(k)\right\rangle_{B},
\end{aligned}
$$

and the backward infinitesimal generator

$$
\begin{aligned}
\mathcal{L}_{\eta_{t}^{*}}^{*} f(k)= & \langle c, \nabla f(k)\rangle-\frac{1}{2} \Delta_{B} f(k)-\int_{\mathbb{R}^{d}}\left(f(k-y)-f(k)+\langle y, \nabla f(k)| 1_{\{|y| \leqslant 1\}} \frac{\eta_{t}^{*}(k-y)}{\eta_{t}^{*}(k)} v(d y)\right. \\
& +\int_{\mathbb{R}^{d}} \frac{\eta_{t}^{*}(k-y)-\eta_{t}^{*}(k)}{\eta_{t}^{*}(k)}\langle y, \nabla f(k)| 1_{\{|y| \leqslant 1\}} v(d y)-\left\langle\nabla \log \eta_{t}^{*}(k), \nabla f(k)\right\rangle_{B} .
\end{aligned}
$$

With the notation of Section 3 we have $\mathcal{L}_{\eta_{t}^{*}}^{*} \tilde{f}(k)=-\mathcal{L}_{\eta_{t}^{*}(-)} f(-k)=-\mathcal{L}_{\tilde{\eta}_{v-(t-t)}} f(-k)$ and $\mathcal{L}_{\eta_{t}} \tilde{f}(k)=$ $-\mathcal{L}_{\eta_{t}(-.)}^{*} f(-k)=-\mathcal{L}_{\tilde{\eta}_{v-(t-t)}^{*}}^{*} f(-k)$, hence $-\mathcal{L}_{\eta_{t}^{*}}^{*}$ and $-\mathcal{L}_{\eta_{t}}$ are respectively the forward and backward generators of the reversed process $\left(-\tilde{z}_{v-(t-r)}\right)_{t \in[r, v]}$. If $v$ is symmetric then $\mathcal{L}_{\eta_{t}^{*}}^{*}=-\mathcal{L}_{\tilde{\eta}_{v-(t-t)}} \mathcal{L}_{\eta_{t}}=-\mathcal{L}_{\tilde{\eta}_{v-(t-t)}^{*}}^{*}$, and the process $\left(z_{t}\right)_{t \in[r, v]}$ is reversible provided its initial and final laws $\pi_{r}, \pi_{v}$ are symmetric. The knowledge of the generators of $\left(z_{t}\right)_{t \in[r, v]}$ provides the forward and backward representations of $\left(z_{t}\right)_{t \in[r, v]}$ as weak solutions of stochastic integro-differential equations. We assume that (cf. p. 434 of [22]):

(A) the functions

$$
\begin{aligned}
& (t, k) \mapsto \int_{\mathbb{R}^{d}}\left(1 \wedge|y|^{2}\right) \frac{\eta_{t}(k+y)}{\eta_{t}(k)} v(d y), \\
& (t, k) \mapsto \int_{\{|y| \leqslant 1\}} y \frac{\eta_{t}(k+y)-\eta_{t}(k)}{\eta_{t}(k)} v(d y), \\
& (t, k) \mapsto \nabla \log \eta_{t}(k),
\end{aligned}
$$

respectively

$$
\begin{aligned}
& (t, k) \mapsto \int_{\mathbb{R}^{d}}\left(1 \wedge|y|^{2}\right) \frac{\eta_{t}^{*}(k-y)}{\eta_{t}^{*}(k)} v(d y), \\
& (t, k) \mapsto \int_{\{|y| \leqslant 1\}} y \frac{\eta_{t}^{*}(k-y)-\eta_{t}^{*}(k)}{\eta_{t}^{*}(k)} v(d y), \\
& (t, k) \mapsto \nabla \log \eta_{t}^{*}(k),
\end{aligned}
$$

are bounded on compacts of $\mathbb{R}_{+} \times \mathbb{R}^{d}$.

The next proposition is a representation result that follows from Proposition 3.4 and Theorems 13.58, 14.80 of [22], pp. 438 and 481, using the results on martingale problems for discontinuous processes of $[25,26,30]$.

Proposition 3.5. The process $\left(z_{t}\right)_{t \in[r, v]}$ of Theorem 3.2 is solution, in the weak sense and with respect to the forward filtration $\left(\mathcal{P}_{t}\right)_{t \in[r, v]}$, of 


$$
\begin{aligned}
d z_{t}= & c d t+d W_{t}+\int_{\mathbb{R}^{d}} y\left(\mu(d y, d t)-\frac{\eta_{t}\left(z_{t^{-}}+y\right)}{\eta_{t}\left(z_{t^{-}}\right)} 1_{\{|y| \leqslant 1\}} v(d y) d t\right) \\
& +\int_{\mathbb{R}^{d}} y \frac{\eta_{t}\left(z_{t^{-}}+y\right)-\eta_{t}\left(z_{t^{-}}\right)}{\eta_{t}\left(z_{t^{-}}\right)} 1_{\{|y| \leqslant 1\}} v(d y) d t+B \nabla \log \eta_{t}\left(z_{t^{-}}\right) d t,
\end{aligned}
$$

under a probability $P$ for which $W_{t}$ is a (forward) Brownian motion with covariance $B$, and $\mu(d y, d s)$ is the canonical point process with compensator $\frac{\eta_{t}\left(z_{t}-y\right)}{\eta_{t}\left(z_{t^{-}}\right)} v(d y) d t$. In terms of backward differentials we have as well, with respect to the decreasing filtration $\left(\mathcal{F}_{t}\right)_{t \in[r, v]}$,

$$
\begin{aligned}
d_{*} z_{t}= & c d t+d_{*} W_{t}^{*}+\int_{\mathbb{R}^{d}} y\left(\mu_{*}(d y, d t)-\frac{\eta_{t}^{*}\left(z_{t^{+}}-y\right)}{\eta_{t}^{*}\left(z_{t^{+}}\right)} 1_{\{|y| \leqslant 1\}} v(d y) d t\right) \\
& +\int_{\mathbb{R}^{d}} y \frac{\eta_{t}^{*}\left(z_{t^{+}}-y\right)-\eta_{t}^{*}\left(z_{t^{+}}\right)}{\eta_{t}^{*}\left(z_{t^{+}}\right)} 1_{\{|y| \leqslant 1\}} v(d y) d t-B \nabla \log \eta_{t}^{*}\left(z_{t^{+}}\right) d t,
\end{aligned}
$$

where $W_{t}^{*}$ denotes a backward Brownian motion with covariance $B$, and $\mu_{*}(d y, d t)$ is the backward Poisson random measure with compensator $\frac{\eta_{t}^{*}\left(z_{t}-y\right)}{\eta_{t}^{*}\left(z_{t^{-}}\right)} \nu(d y) d t$.

This also provides the $\left(\mathcal{P}_{t}\right)_{t \in[r, v]}$-decomposition

$$
z_{t}=z_{r}+c(t-r)+M_{t}+\int_{r}^{t} \int_{\mathbb{R}^{d}} y\left(\frac{\eta_{s}\left(z_{s^{-}}+y\right)}{\eta_{s}\left(z_{s^{-}}\right)}-1_{\{|y| \leqslant 1\}}\right) v(d y) d s+\int_{r}^{t} B \nabla \log \eta_{s}\left(z_{s^{-}}\right) d s,
$$

where $\left(M_{t}\right)_{t \in[0, v]}$ is a $\left(\mathcal{P}_{t}\right)_{t \in[r, v]}$-martingale, and the $\left(\mathcal{F}_{t}\right)_{t \in[r, v] \text {-decomposition }}$

$$
z_{t}=z_{v}-c(v-t)+M_{t}^{*}+\int_{t}^{v} \int_{\mathbb{R}^{d}} y\left(\frac{\eta_{s}^{*}\left(z_{s^{+}}-y\right)}{\eta_{s}^{*}\left(z_{s^{+}}\right)}-1_{\{|y| \leqslant 1\}}\right) v(d y) d s-\int_{t}^{v} B \nabla \log \eta_{s}^{*}\left(z_{s^{+}}\right) d s,
$$

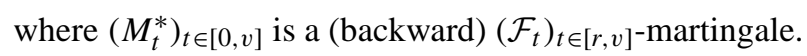

Examples (and particular cases of Proposition 3.5).

(1) Deterministic process.

In this case $\left(z_{t}\right)_{t \in[r, v]}$ satisfy the ordinary differential equation

$$
d z_{t}=d \xi_{t}=c d t
$$

both in the forward and backward cases, hence

$$
z_{t}=z_{r}+c(t-r)=z_{v}+c(v-t), \quad r<t<v,
$$

with (random) initial and final conditions $z_{r}, z_{v}$. The influence of $U$ manifests itself in the initial and final laws, not in the dynamics.

(2) Lévy bridges from $a \in \mathbb{R}^{d}$ to $b \in \mathbb{R}^{d}$.

Take $U=0$, and assume that $\mu_{t}(d k)=\mu_{t}(k) \lambda(d k), t>0$, has a density with respect to a fixed reference measure $\lambda$ for all $t \geqslant 0$. The forward stochastic integro-differential equation satisfied by $\left(z_{t}\right)_{t \in[r, v]}$ is 


$$
\begin{aligned}
d z_{t}= & c d t+d W_{t}+\int_{\mathbb{R}^{d}} y\left(\mu(d y, d t)-\frac{\mu_{t-v}\left(z_{t^{-}}+y-b\right)}{\mu_{t-v}\left(z_{t^{-}}-b\right)} 1_{\{|y| \leqslant 1\}} v(d y) d t\right) \\
& +\int_{\mathbb{R}^{d}} y \frac{\mu_{t-v}\left(z_{t^{-}}+y-b\right)-\mu_{t-v}\left(z_{t^{-}}-b\right)}{\mu_{t-v}\left(z_{t^{-}}-b\right)} 1_{\{|y| \leqslant 1\}} v(d y) d t-B \nabla \log \mu_{t-v}\left(z_{t^{-}}-b\right) d t,
\end{aligned}
$$

i.e. using Lemma 2.2:

$$
d z_{t}=d W_{t}+\int_{\mathbb{R}^{d}} y\left(\mu(d y, d t)-\frac{\mu_{t-v}\left(z_{t^{-}}+y-b\right)}{\mu_{t-v}\left(z_{t^{-}}-b\right)} v(d y) d t\right)-\frac{z_{t^{-}}-b}{v-t} d t .
$$

From this equation it follows that the expectation $\left(E\left[z_{t}\right]\right)_{t \in[r, v]}$ of a Lévy bridge always satisfies the ordinary differential equation

$$
\frac{d}{d t} E\left[z_{t}\right]=\frac{b-E\left[z_{t}\right]}{v-t}, \quad r \leqslant t \leqslant v,
$$

with initial condition $E\left[z_{r}\right]=a$, i.e. the expectation of $z_{t}$ follows the linear interpolation

$$
E\left[z_{t}\right]=a+(b-a) \frac{t-r}{v-r}, \quad r \leqslant t \leqslant v,
$$

between $(r, a)$ and $(v, b)$, which is a particular case of the harness property [16,12]. The backward stochastic differential equation satisfied by the same process $\left(z_{t}\right)_{t \in[r, v]}$ is:

$$
\begin{aligned}
d_{*} z_{t}= & c d t+d_{*} W_{t}^{*}+\int_{\mathbb{R}^{d}} y\left(\mu_{*}(d y, d t)-\frac{\mu_{t-r}\left(z_{t^{+}}+y-a\right)}{\mu_{t-r}\left(z_{t^{+}}-a\right)} 1_{\{|y| \leqslant 1\}} v(d y) d t\right) \\
& +\int_{\mathbb{R}^{d}} y \frac{\mu_{t-r}\left(z_{t^{+}}+y-a\right)-\mu_{t-r}\left(z_{t^{+}}-a\right)}{\mu_{t-r}\left(z_{t^{+}}-a\right)} 1_{\{|y| \leqslant 1\}} v(d y) d t-B \nabla \log \mu_{t-r}\left(z_{t^{+}}-a\right) d t,
\end{aligned}
$$

i.e. by Lemma 2.2:

$$
d_{*} z_{t}=d_{*} W_{t}^{*}+\int_{\mathbb{R}^{d}} y\left(\mu_{*}(d y, d t)-\frac{\mu_{t-r}\left(z_{t^{+}}+y-a\right)}{\mu_{t-r}\left(z_{t^{+}}-a\right)} v(d y) d t\right)+\frac{z_{t^{+}}-a}{t-r} d t .
$$

(3) Poisson bridge starting at $a \in \mathbb{N}$ at time $r$ and ending at $b \in \mathbb{N}$ at time $v$.

If $U=0$, the forward (i.e. $\left(\mathcal{P}_{t}\right)_{t \in[r, v]^{-}}$) stochastic equation (3.1) satisfied by the Poisson bridge is written

$$
d z_{t}=d N_{t}^{\eta}, \quad z_{r}=a,
$$

where $\left(N_{t}^{\eta}\right)_{t \in[r, v]}$ is a point process starting from 0 at time $r$, with compensator

$$
d\left\langle N_{t}^{\eta}\right\rangle=\frac{\eta_{t}\left(z_{t^{-}}+1\right)}{\eta_{t}\left(z_{t^{-}}\right)} d t=\frac{\mu_{v-t}\left(b-z_{t^{-}}-1\right)}{\mu_{v-t}\left(b-z_{t^{-}}\right)} d t=\frac{b-a-N_{t^{-}}^{\eta}}{v-t} d t .
$$

This means (see e.g. Theorem 7.4. p. 93 of [20] and references therein) that $\left(z_{t}\right)_{t \in[r, v]}$ can be constructed by a time change on a standard Poisson process $(N(t))_{t \in \mathbb{R}_{+}}$, i.e. the sequence of jump times $\left(T_{k}^{\eta}\right)_{1 \leqslant k \leqslant m-i}$ of $\left(z_{t}\right)_{t \in[r, v]}=\left(a+N_{t}^{\eta}\right)_{t \in[r, v]}$ can be obtained by induction from the jump times $\left(T_{k}\right)_{k} \geqslant 1$ of $(N(t))_{t \in \mathbb{R}_{+}}$, as

$$
T_{k}=\sum_{i=1}^{k} \int_{T_{i-1}^{\eta}}^{T_{i}^{\eta}} \frac{b-a-(i-1)}{v-s} d s, \quad 1 \leqslant k \leqslant b-a .
$$


The backward equation satisfied by $\left(z_{t}\right)_{t \in[r, v]}$ is

$$
d_{*} z_{t}=d_{*} N_{t}^{\eta^{*}}, \quad z_{v}=b,
$$

where $\left(N_{t}^{\eta^{*}}\right)_{t \in[r, v]}=\left(-N_{v-t}^{\eta}\right)_{t \in[r, v]}$ is a point process starting from 0 at time $v$, with backward compensator

$$
d_{*}\left\langle N_{t}^{\eta^{*}}\right\rangle=\frac{\eta_{t}^{*}\left(z_{t^{+}}-1\right)}{\eta_{t}^{*}\left(z_{t^{+}}\right)} d t=-\frac{N_{t^{+}}^{\eta^{*}}+b-a}{r-t} d t .
$$

(4) Gamma bridge.

The compensator is given for $k \leqslant b$ by:

$$
\int_{\mathbb{R}} y \frac{\mu_{t-v}(k+y-b)}{\mu_{t-v}(k-b)} 1_{\{|y| \leqslant 1\}} v(d y)=\int_{0}^{k-b}\left(\frac{b-k-y}{b-k}\right)^{v-t-1} d y=-\frac{k-b}{v-t},
$$

which coincides with the general drift found in (3.11).

(5) Brownian bridge.

We have $\mu_{t}(k)=\frac{1}{\sqrt{2 \pi t}} e^{-k^{2} /(2 t)}$, hence the forward and backward stochastic differential equations satisfied by $\left(z_{t}\right)_{t \in[r, v]}$ are

$$
d z_{t}=d W_{t}-\frac{z_{t}-b}{v-t} d t, \quad z_{r}=a,
$$

and

$$
d_{*} z_{t}=d_{*} W_{t}^{*}+\frac{z_{t}-a}{t-r} d t, \quad z_{v}=b .
$$

(6) Forward Lévy processes $(U=0)$.

Assuming that $\mu_{t}(d k)=\mu_{t}(k) \lambda(d k)$ is absolutely continuous with respect to $\lambda(d k)$ we have

$$
d z_{t}=c d t+d W_{t}+\int_{\mathbb{R}^{d}} y\left(\mu(d y, d t)-1_{\{|y| \leqslant 1\}} v(d y) d t\right), \quad z_{r}=\xi_{r},
$$

i.e. $z_{t}=\xi_{t}, r<t<v$. Besides the forward generator $-V(i \nabla)$ of $\left(\xi_{t}\right)_{t \in[r, v]}$ (see (2.1)) we obtain the backward generator

$$
\begin{aligned}
\mathcal{L}_{\eta_{t}^{*}}^{*} f(k)=\langle c & , \nabla f(k)\rangle-\frac{1}{2} \Delta_{B} f(k)-\int_{\mathbb{R}^{d}}\left(f(k-y)-f(k)+\langle y, \nabla f(k)| 1_{\{|y| \leqslant 1\}}\right) \frac{\mu_{t}(k-y)}{\mu_{t}(k)} v(d y) \\
& +\int_{\mathbb{R}^{d}} \frac{\mu_{t}(k-y)-\mu_{t}(k)}{\mu_{t}(k)}\langle y, \nabla f(k)| 1_{\{|y| \leqslant 1\}} v(d y)-\left\langle\nabla \log \mu_{t}(k), \nabla f(k)\right\rangle_{B},
\end{aligned}
$$

or by Lemma 2.2 above:

$$
\mathcal{L}_{\eta_{t}^{*}}^{*} f(k)=-\frac{1}{2} \Delta_{B} f(k)-\int_{\mathbb{R}^{d}}(f(k-y)-f(k)+\langle y, \nabla f(k)\rangle) \frac{\mu_{t}(k-y)}{\mu_{t}(k)} v(d y)+\frac{1}{t}\langle k, \nabla f(k)\rangle .
$$

The backward stochastic differential equation satisfied by $\left(\xi_{t}\right)_{t \in[r, v]}$ is:

$$
\begin{aligned}
d_{*} z_{t}= & c d t+d_{*} W_{t}^{*}+\int_{\mathbb{R}^{d}} y\left(\mu_{*}(d y, d t)-\frac{\mu_{t}\left(z_{t^{+}}-y\right)}{\mu_{t}\left(z_{t^{+}}\right)} 1_{\{|y| \leqslant 1\}} v(d y) d t\right) \\
& +\int_{\mathbb{R}^{d}} y \frac{\mu_{t}\left(z_{t^{+}}-y\right)-\mu_{t}\left(z_{t^{+}}\right)}{\mu_{t}\left(z_{t^{+}}\right)} 1_{\{|y| \leqslant 1\}} v(d y) d t-B \nabla \log \mu_{t}\left(z_{t^{+}}\right) d t,
\end{aligned}
$$


i.e. from Lemma 2.2:

$$
d_{*} z_{t}=d_{*} W_{t}^{*}+\int_{\mathbb{R}^{d}} y\left(\mu_{*}(d y, d t)-\frac{\mu_{t}\left(z_{t^{+}}-y\right)}{\mu_{t}\left(z_{t^{+}}\right)} v(d y) d t\right)+\frac{z_{t^{+}}}{t} d t .
$$

In other terms we have the backward martingale decomposition

$$
z_{t}=M_{t}^{*}+\int_{t}^{v} \frac{z_{s}}{s} d s, \quad r<t<v
$$

in which $M_{t}^{*}$ denotes the backward martingale part, recovering and extending some results in [23].

(7) Backward Lévy processes.

Taking $\eta_{v}(d m)=\mu_{0}(m) \lambda(d m)=\mu_{0}(d m)$ and $\eta_{r}^{*}(i)=1$, we have $\eta_{t}(k)=\eta_{v} * \mu_{t-v}(k)=\mu_{t-v}(k)=$ $\mu_{v-t}(-k)$, and $\eta_{t}^{*}(k)=1, r<t<v$, hence $\left(z_{t}\right)_{t \in[0, v]}$ is the backward Lévy process given by

$$
d_{*} z_{t}=c d t+d_{*} W_{t}^{*}+\int_{\mathbb{R}^{d}} y\left(\mu_{*}(d y, d t)-1_{\{|y| \leqslant 1\}} v(d y) d t\right),
$$

which has same law as the time reversed Lévy process $\left(-\xi_{s}^{*}\right)_{s \in[0, v]}=\left(-\xi_{v-s}\right)_{s \in[0, v]}$. The forward generator of $\left(z_{t}\right)_{t \in[0, v]}$ is

$$
\begin{aligned}
\mathcal{L}_{\eta_{t}} f(k)= & \langle c, \nabla f(k)\rangle+\frac{1}{2} \Delta_{B} f(k)+\int_{\mathbb{R}^{d}}\left(f(k+y)-f(k)-\langle y, \nabla f(k)| 1_{\{|y| \leqslant 1\}}\right) \frac{\mu_{t-v}(k+y)}{\mu_{t-v}(k)} v(d y) \\
& +\int_{\mathbb{R}^{d}} \frac{\mu_{t-v}(k+y)-\mu_{t-v}(k)}{\mu_{t-v}(k)}\langle y, \nabla f(k)| 1_{\{|y| \leqslant 1\}} v(d y)+\left\langle\nabla \log \mu_{t-v}(k), \nabla f(k)\right\rangle_{B},
\end{aligned}
$$

or from Lemma 2.2:

$$
\mathcal{L}_{\eta_{t}} f(k)=\frac{1}{2} \Delta_{B} f(k)+\int_{\mathbb{R}^{d}}(f(k+y)-f(k)-\langle y, \nabla f(k)\rangle) \frac{\mu_{t-v}(k+y)}{\mu_{t-v}(k)} v(d y)-\frac{1}{v-t}\langle k, \nabla f(k)\rangle .
$$

The forward stochastic differential equation satisfied by $\left(z_{t}\right)_{t \in[0, v]}$ is, therefore,

$$
\begin{aligned}
d z_{t}= & c d t+d W_{t}+\int_{\mathbb{R}^{d}} y\left(\mu(d y, d t)-\frac{\mu_{t-v}\left(z_{t^{-}}+y\right)}{\mu_{t-v}\left(z_{t^{-}}\right)} 1_{\{|y| \leqslant 1\}} v(d y) d t\right) \\
& -\int_{\mathbb{R}^{d}} y \frac{\mu_{t-v}\left(z_{t^{-}}\right)-\mu_{t-v}\left(z_{t^{-}}+y\right)}{\mu_{t-v}\left(z_{t^{-}}\right)} 1_{\{|y| \leqslant 1\}} v(d y) d t+B \nabla \log \mu_{t-v}\left(z_{t^{-}}\right) d t,
\end{aligned}
$$

where $W_{t}$ is a forward Brownian motion with covariance $B$, and $\mu(d y, d t)$ is the forward Poisson random measure with compensator $\frac{\mu_{t-v}\left(z_{t}+y\right)}{\mu_{t-v}\left(z_{t}-\right)}$, i.e. by Lemma 2.2:

$$
d z_{t}=d W_{t}+\int_{\mathbb{R}^{d}} y\left(\mu(d y, d t)-\frac{\mu_{t-v}\left(z_{t^{-}}+y\right)}{\mu_{t-v}\left(z_{t^{-}}\right)} v(d y) d t\right)-\frac{z_{t^{-}}}{v-t}
$$

and we have the forward martingale decomposition

$$
z_{t}=M_{t}-\int_{r}^{t} \frac{z_{s}}{v-s} d s, \quad r<t<v,
$$


to be compared to [23] (note that here we took $z_{v}=0$ ). The forward compensator of $\left(z_{t}\right)_{t \in[0, v]}$ is again

$$
d\left\langle z_{t}\right\rangle=-\frac{z_{t^{-}}}{v-t} d t
$$

\section{Girsanov theorem}

The next proposition shows that under certain conditions, the law of the process $\left(z_{t}\right)_{t \in[r, v]}$ of Proposition 3.1 is absolutely continuous with respect to the law of the Lévy process $\left(\xi_{t}\right)_{t \in[r, v]}$.

Proposition 4.1. Assume that $c=0, v(\{|y| \geqslant 1\})=0$ and either $B=0$ or $v=0$, i.e. we are in the Brownian case or in the jump case. Under the hypothesis of Theorem 3.2, the law $Q$ of $\left(z_{t}\right)_{t \in[r, v]}$ is absolutely continuous with respect to $P$, with density given by

$$
\left.\frac{d Q}{d P}\right|_{\mathcal{P}_{t}}=\frac{\eta_{t}\left(z_{t}\right)}{\eta_{r}\left(z_{r}\right)} e^{-\int_{r}^{t} U\left(z_{\tau}\right) d \tau}, \quad r \leqslant t \leqslant v,
$$

i.e. under $Q,\left(z_{r}+\xi_{t}\right)_{t \in[r, v]}$ has the law of $\left(z_{t}\right)_{t \in[r, v]}$ under P. Similarly we have

$$
\left.\frac{d Q}{d P}\right|_{\mathcal{F}_{t}}=\frac{\eta_{t}^{*}\left(z_{t}\right)}{\eta_{v}^{*}\left(z_{v}\right)} e^{-\int_{t}^{v} U\left(z_{\tau}\right) d \tau}, \quad r \leqslant t \leqslant v,
$$

i.e. $\left(z_{v}-\xi_{v-t}\right)_{t \in[r, v]}$ has the law of $\left(z_{t}\right)_{t \in[r, v]}$ under $P$.

Proof. Let us define

$$
L_{t}=\frac{\eta_{t}\left(z_{t}\right)}{\eta_{r}\left(z_{r}\right)} e^{-\int_{r}^{t} U\left(z_{\tau}\right) d \tau}, \quad t \in[r, v] .
$$

Assume that under $P, \mu(d y, d t)$ is the random measure with compensator $v(d y) d t$ in the Poisson case, with

$$
d z_{t}=\int_{\mathbb{R}^{d}} y\left(\mu(d y, d t)-1_{\{|y| \leqslant 1\}} v(d y) d t\right),
$$

or that $\left(z_{t}\right)_{t \in[r, v]}$ is a standard Brownian motion in the Brownian case, with $d W_{t}=d z_{t}-B \nabla \log \eta_{t}\left(z_{t^{-}}\right) d t$, i.e.

$$
d z_{t}=d W_{t}+B \nabla \log \eta_{t}\left(z_{t^{-}}\right) d t .
$$

Let us compute

$$
\begin{aligned}
d \eta_{t}\left(z_{t}\right)= & \eta_{t}\left(z_{t^{-}}\right) \int_{|y| \leqslant 1} \frac{\eta_{t}\left(z_{t^{-}}+y\right)-\eta_{t}\left(z_{t^{-}}\right)}{\eta_{t}\left(z_{t^{-}}\right)}\left(\mu(d y, d t)-\frac{\eta_{t}\left(z_{t^{-}}+y\right)}{\eta_{t}\left(z_{t^{-}}\right)} v(d y) d t\right) \\
& +\eta_{t}\left(z_{t^{-}}\right)\left\langle\nabla \log \eta_{t}\left(z_{t^{-}}\right), d W_{t}\right\rangle_{B}+\mathcal{L}_{\eta_{t}} \eta_{t}\left(z_{t}\right) d t+\frac{\partial \eta_{t}}{\partial t}\left(z_{t}\right) d t \\
= & U\left(z_{t^{-}}\right) \eta_{t}\left(z_{t^{-}}\right) d t+\eta_{t}\left(z_{t^{-}}\right) \int_{|y| \leqslant 1} \frac{\eta_{t}\left(z_{t^{-}}+y\right)-\eta_{t}\left(z_{t^{-}}\right)}{\eta_{t}\left(z_{t^{-}}\right)}(\mu(d y, d t)-v(d y) d t) \\
& +\eta_{t}\left(z_{t^{-}}\right)\left\langle\nabla \log \eta_{t}\left(z_{t^{-}}\right), d W_{t}\right\rangle_{B}+\left\langle\nabla \log \eta_{t}\left(z_{t^{-}}\right), \nabla \eta_{t}\left(z_{t^{-}}\right)\right\rangle_{B},
\end{aligned}
$$

where we used (6.1) and the forward infinitesimal generator 


$$
\begin{aligned}
\mathcal{L}_{\eta_{t}} \eta_{t}(k)= & -H \eta_{t}(k)+U(k) \eta_{t}(k)+\left\langle\nabla \log \eta_{t}(k), \nabla \eta_{t}(k)\right\rangle_{B} \\
& +\int_{\mathbb{R}^{d}}\left(\frac{\eta_{t}^{2}(k+y)}{\eta_{t}(k)}-\eta_{t}(k)\right) v(d y) d t+\frac{\partial \eta_{t}}{\partial t}\left(z_{t}\right) .
\end{aligned}
$$

Hence $\left(L_{t}\right)_{t \in[r, v]}$ satisfies the (forward) stochastic integro-differential equation

$$
\begin{aligned}
d L_{t}= & L_{t^{-}} \cdot \int_{|y| \leqslant 1} \frac{\eta_{t}\left(z_{t^{-}}+y\right)-\eta_{t}\left(z_{t^{-}}\right)}{\eta_{t}\left(z_{t^{-}}\right)}(\mu(d y, d t)-v(d y) d t) \\
& +L_{t^{-}} \cdot\left\langle\nabla \log \eta_{t}\left(z_{t^{-}}\right), d W_{t}+\nabla \log \eta_{t}\left(z_{t^{-}}\right) d t\right\rangle_{B}, \quad t \in[r, v] .
\end{aligned}
$$

Under $P$ we have

$$
\frac{1}{L_{s^{-}}} d\left\langle L_{s}, z_{s}\right\rangle=\int_{|y| \leqslant 1} \frac{\eta_{t}\left(z_{t^{-}}+y\right)-\eta_{t}\left(z_{t^{-}}\right)}{\eta_{t}\left(z_{t^{-}}\right)} v(d y) d t+B \nabla \log \eta_{t}\left(z_{t^{-}}\right),
$$

and from the Girsanov theorem, for $t \in[r, v]$,

$$
\begin{aligned}
z_{t}-\int_{r}^{t} \frac{1}{L_{s^{-}}} d\left\langle L_{s}, z_{s}\right\rangle= & W_{t}-W_{r}+\int_{r}^{t} B \nabla \log \eta_{s}\left(z_{s^{-}}\right) d s \\
& +\int_{r}^{t} \int_{\{|y| \leqslant 1\}} y(\mu(d y, d s)-v(d y) d s)-\int_{r}^{t} \frac{1}{L_{s^{-}}} d\left\langle L_{s}, z_{s}\right\rangle \\
= & W_{t}+\int_{r}^{t} \int_{\{|y| \leqslant 1\}} y\left(\mu(d y, d s)-\frac{\eta_{s}\left(z_{s^{-}}+y\right)}{\eta_{s}\left(z_{s^{-}}\right)} v(d y) d s\right),
\end{aligned}
$$

is a $\left(\mathcal{P}_{t}\right)_{t \in[r, v]}$-martingale under the probability $Q$ defined by

$$
\left.\frac{d Q}{d P}\right|_{\mathcal{P}_{t}}=L_{t}, \quad r \leqslant t \leqslant v,
$$

hence under $Q, W_{t}$ is a Brownian motion (in the Brownian case) and $\mu(d y, d s)$ has the compensator $\frac{\eta_{s}\left(z_{s}+y\right)}{\eta_{s}\left(z_{s}-\right)} v(d y)$ (in the pure jump case), i.e. $\left(z_{r}+\xi_{t-r}\right)_{t \in[r, v]}$ has the law of $\left(z_{t}\right)_{t \in[r, v]}$ under $P$.

The proof in the backward case is similar.

\section{Examples (for Proposition 4.1).}

(1) Deterministic process. In this case we have $Q=P, \eta_{t}\left(z_{t}\right)=\eta_{r}\left(z_{r}\right), \eta_{t}^{*}\left(z_{t}\right)=\eta_{v}^{*}\left(z_{v}\right), r \leqslant t \leqslant v$, and, in fact, $\left(z_{t}\right)_{t \in[r, v]}=\left(\xi_{t}\right)_{t \in[r, v] \text {. }}$

(2) Lévy bridges from $a \in \mathbb{R}^{d}$ to $b \in \mathbb{R}^{d}$.

Take $U=0$, and assume that $\mu_{t}(d k)=\mu_{t}(k) \lambda(d k)$ has a density with respect to $\lambda$. We have

$$
\left.\frac{d Q}{d P}\right|_{\mathcal{P}_{t}}=\frac{\mu_{v-t}\left(b-z_{t}\right)}{\mu_{v}\left(b-z_{r}\right)}, \quad \text { and }\left.\quad \frac{d Q}{d P}\right|_{\mathcal{F}_{t}}=\frac{\mu_{t-r}\left(z_{t}-a\right)}{\mu_{v-r}\left(z_{v}-a\right)}, \quad r \leqslant t \leqslant v .
$$

(3) Poisson bridge from $a \in \mathbb{N}$ to $b \in \mathbb{N}, a \leqslant b$. 
In this case the law of $\left(z_{t}\right)_{t \in[r, v]}$ is absolutely continuous with respect to $P$, with

$$
\left.\frac{d Q}{d P}\right|_{\mathcal{P}_{t}}=\frac{\eta_{t}\left(z_{t}\right)}{\eta_{r}\left(z_{r}\right)}=e^{t-r} \frac{(v-t)^{b-z_{t}}}{(v-r)^{b-a}} \frac{(b-a) !}{\left(b-z_{t}\right) !} 1_{[a, b]}\left(z_{t}\right), \quad r \leqslant t \leqslant v,
$$

hence

$$
\frac{d Q}{d P}=e^{v-r} \frac{(b-a) !}{(v-r)^{b-a}} 1_{\left\{z_{v}=b\right\}},
$$

with $z_{r}=a$, i.e. under $Q$, the standard Poisson process $\left(a+\xi_{t-r}\right)_{t \in[r, v]}$ has the law of the Poisson bridge

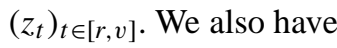

$$
\left.\frac{d Q}{d P}\right|_{\mathcal{F}_{t}}=\frac{\eta_{t}^{*}\left(z_{t}\right)}{\eta_{v}^{*}\left(z_{v}\right)}=e^{-(t-v)} \frac{(t-r)^{z_{t}}(b-a) !}{z_{t} !(v-r)^{(b-a)}} 1_{[a, b]}\left(z_{t}\right), \quad r \leqslant t \leqslant v,
$$

hence

$$
\frac{d Q}{d P}=e^{-(r-v)} \frac{(b-a) !}{(v-r)^{(b-a)}} 1_{\{z r=a\}},
$$

with $z_{v}=b$, i.e. under $Q,\left(b-\xi_{v-t}\right)_{t \in[r, v]}$ has the law of the Poisson bridge.

(4) Brownian and gamma bridges.

The law of the Brownian bridge starting from $a \in \mathbb{R}$ at time $r$ and ending at $b \in \mathbb{R}$ is not absolutely continuous with respect to the Wiener measure, since $\mu_{r}(d i)=\delta_{a}(d i)$ and $\mu_{v}(d m)=\delta_{b}(d m)$. The same holds for the gamma bridge.

(5) Forward and backward Brownian motion $(U=0)$.

We have either $Q=P$ or $Q$ is not absolutely continuous with respect to $P$.

(6) Forward and backward Poisson processes $(U=0)$.

In the standard Poisson case, backward Lévy processes give examples of jump processes with $z_{v}=0$ and initial Poisson distribution $\rho_{r}(k) \lambda(d k), k \leqslant 0$, on $-\mathbb{N}$. We have $\mu_{t-v}(k)=e^{-(v-t)}(v-t)^{-k} /(-k) !, t \leqslant v$, and

$$
\left.\frac{d Q}{d P}\right|_{\mathcal{P}_{t}}=\frac{\mu_{t-v}\left(z_{t}\right)}{\mu_{r-v}\left(z_{r}\right)}=e^{-(r-t)} \frac{(v-t)^{-z_{t}}}{\left(-z_{t}\right) !} \frac{\left(-z_{r}\right) !}{(v-r)^{-z_{r}}},
$$

hence

$$
\frac{d Q}{d P}=e^{v-r} \frac{\left(-z_{r}\right) !}{(v-r)^{-z_{r}}} 1_{\left\{z_{v}=0\right\}} .
$$

It follows from Proposition 4.1 that under $Q$ the process $\left(z_{r}+\xi_{t-r}\right)_{t \in[r, v]}$ has the law of $\left(z_{t}\right)_{t \in[r, v]}$, where $\left(\xi_{t}\right)_{t \in[0,+\infty[}$ is the canonical Lévy process. Similarly we have, if $\left(z_{t}\right)_{t \in[r, v]}$ is a standard Poisson process under $P$ :

$$
\left.\frac{d Q}{d P}\right|_{\mathcal{F}_{t}}=\frac{\mu_{t}\left(z_{t}\right)}{\mu_{v}\left(z_{v}\right)}=e^{-(t-v)} \frac{(t-r)^{z_{t}}}{z_{t} !} \frac{z_{v} !}{(v-r)^{z_{v}}}
$$

hence

$$
\frac{d Q}{d P}=e^{v-r} \frac{z_{v} !}{(v-r)^{z_{v}}} 1_{\left\{z_{r}=0\right\}}, \quad r \geqslant 0,
$$

i.e. under $Q,\left(z_{v}-\xi_{t}^{*}\right)_{t \in[r, v]}=\left(z_{v}-\xi_{v-t}\right)_{t \in[r, v]}$ has same the law as the standard (forward) Poisson process $\left(\xi_{t}\right)_{t \in[r, v] \text {. }}$

(7) Forward and backward Lévy processes ( $U=0$ and $\mu_{r}, \mu_{v}$ are absolutely continuous with respect to $\lambda$ ). 
Here the probability $Q$ is naturally equal to $P$, and the process $\left(z_{t}\right)_{t \in[r, v]}$ has same law as the forward Lévy

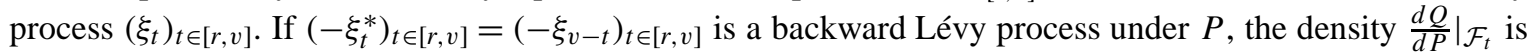
given by

$$
\left.\frac{d Q}{d P}\right|_{\mathcal{F}_{t}}=\frac{\mu_{t}\left(z_{t}\right)}{\mu_{v}\left(z_{v}\right)}
$$

and

$$
\frac{d Q}{d P}=\frac{\mu_{r}\left(z_{r}\right)}{\mu_{v}\left(z_{v}\right)},
$$

i.e. $\left(z_{v}-\xi_{t}^{*}\right)_{t \in[r, v]}\left(z_{v}-\xi_{v-t}\right)_{t \in[r, v]}$ is a forward Lévy process under $Q$. Similarly, the process $\left(z_{t}\right)_{t \in[r, v]}$ has same law as the backward Lévy process $\left(-\xi_{t}^{*}\right)_{t \in[r, v]}=\left(-\xi_{t-v}\right)_{t \in[r, v]}$. The density $\left.\frac{d Q}{d P}\right|_{\mathcal{P}_{t}}$ is given by

$$
\left.\frac{d Q}{d P}\right|_{\mathcal{P}_{t}}=\frac{\mu_{t-v}\left(z_{t}\right)}{\mu_{r-v}\left(z_{r}\right)},
$$

and

$$
\frac{d Q}{d P}=\frac{\mu_{0}\left(z_{v}\right)}{\mu_{r-v}\left(z_{r}\right)} .
$$

From Proposition 4.1, under $Q$ the process $\left(z_{r}+\xi_{t-r}\right)_{t \in[r, v]}$ has the law of the canonical Lévy process $\left(\xi_{t}\right)_{t \in[r, v]}$. [27].

Many of the above examples can be symmetrized (with $H=H^{\dagger}$ ), and can then be interpreted physically, cf.

\section{Reversible diffusion processes with jumps}

In this section we prove Theorem 3.2 and some extensions. This provides a construction of Markovian "bridges" with given initial and final laws since, from Theorem 3.3, $\eta_{r}^{*}$ and $\eta_{v}$ can be chosen so that the products $\eta_{r}^{*} \eta_{r}$ and $\eta_{v}^{*} \eta_{v}$ equal any strictly positive initial and final probability densities fixed in advance. Define the forward and backward Markov transition semi-groups for $s \leqslant r \leqslant t \leqslant u$ and $j, k, l \in \mathbb{R}^{d}$ by:

$$
p(t, k, u, d l)=\frac{\eta_{u}(l)}{\eta_{t}(k)} h(t, k, u, d l),
$$

and

$$
p^{*}(s, d j, t, k)=\frac{\eta_{s}^{*}(j)}{\eta_{t}^{*}(k)} h^{\dagger}(s, d j, t, k) .
$$

The adjointness relation between $H$ and $H^{\dagger}$ :

$$
h(s, j, t, d k) \lambda(d j)=h^{\dagger}(s, d j, t, k) \lambda(d k)
$$

shows that the following reversibility condition holds:

$$
\begin{aligned}
\eta_{s}^{*}(d j) \eta_{s}(j) p(s, j, t, d k) & =\eta_{s}^{*}(d j) h(s, j, t, d k) \eta_{t}(k) \\
& =\eta_{s}^{*}(j) h^{\dagger}(s, d j, t, k) \eta_{t}(d k) \\
& =p^{*}(s, d j, t, k) \eta_{t}^{*}(d k) \eta_{t}(k) .
\end{aligned}
$$


Let us stress that this property generalizes the one understood, since Kolmogorov, as defining the reversibility of a probability measure (cf., for example, [10]). More generally we have

$$
\begin{aligned}
& \eta_{t_{1}}^{*}\left(d k_{1}\right) \eta_{t_{1}}\left(k_{1}\right) p\left(t_{1}, k_{1}, t_{2}, d k_{2}\right) \cdots p\left(t_{n-1}, k_{n-1}, t_{n}, d k_{n}\right) \\
& \quad=\eta_{t_{1}}^{*}\left(d k_{1}\right) h\left(t_{1}, k_{1}, t_{2}, d k_{2}\right) \cdots h\left(t_{n-1}, k_{n-1}, t_{n}, d k_{n}\right) \eta_{t_{n}}\left(k_{n}\right) \\
& \quad=\eta_{t_{1}}^{*}\left(k_{1}\right) h^{\dagger}\left(t_{1}, d k_{1}, t_{2}, k_{2}\right) \cdots h^{\dagger}\left(t_{n-1}, d k_{n-1}, t_{n}, k_{n}\right) \eta_{t_{n}}\left(d k_{n}\right) \\
& \quad=p^{*}\left(t_{1}, k_{1}, t_{2}, d k_{2}\right) \cdots p^{*}\left(t_{n-1}, d k_{n-1}, t_{n}, k_{n}\right) \eta_{t_{n}}^{*}\left(k_{n}\right) \eta_{t_{n}}\left(d k_{n}\right),
\end{aligned}
$$

hence the forward Markov process with transition $p(s, j, t, d k)$ and initial law $\eta_{s}^{*}(d j) \eta_{s}(j)$ has the same law $\eta_{t}^{*}(d k) \eta_{t}(k)$ as the backward Markov process with transition $p^{*}(t, d k, u, l)$ and final law $\eta_{u}^{*}(d l) \eta_{u}(l), s \leqslant t \leqslant u$.

This argument is made precise in the next two propositions, without assuming that $\eta_{r}^{*}(d k)$, respectively $\eta_{v}(d k)$, has a density with respect to $\lambda(d k)$.

Proposition 5.1. Let $\eta_{r}^{*}(d i)$ and $\eta_{v}: \mathbb{R}^{d} \rightarrow \mathbb{R}_{+}$be initial and final conditions such that for some $t \in[r, v]$,

$$
\int_{\mathbb{R}^{d}} \eta_{t}^{*}(d k) \eta_{t}(k)=1
$$

where

$$
\eta_{t}^{*}(d k)=\int_{\mathbb{R}^{d}} \eta_{r}^{*}(d i) h(r, i, t, d k), \quad \eta_{t}(k)=\int_{\mathbb{R}^{d}} \eta_{v}(m) h(t, k, v, d m)=e^{-(v-t) H} \eta_{v}(k),
$$

$r<t<v$, and let us define

$$
p(t, k, u, d l)=\frac{\eta_{u}(l)}{\eta_{t}(k)} h(t, k, u, d l) .
$$

Then

(i) $p(t, k, u, d l)$ is a forward Markov transition kernel,

(ii) the inhomogeneous Markov process $\left(z_{t}\right)_{t \in[r, v]}$ with forward transition kernel $p(t, k, u, d l)$ and initial distribution $\eta_{s}(j) \eta_{s}^{*}(d j)$ satisfies

$$
P\left(z_{t} \in d k \mid \mathcal{P}_{s} \vee \mathcal{F}_{u}\right)=P\left(z_{t} \in d k \mid z_{s}, z_{u}\right), \quad r \leqslant s<t<u \leqslant v,
$$

i.e. it is a Bernstein (or reciprocal, or "local Markov" [8]) process,

(iii) the law at time $t$ of $z_{t}$ is $\rho_{t}(d k)=\eta_{t}(k) \eta_{t}^{*}(d k), t \in[r, v]$.

If moreover $H$ and $H^{\dagger}$ are adjoint with respect to some fixed reference measure $\lambda$ :

$$
h(s, j, t, d k) \lambda(d j)=h^{\dagger}(s, d j, t, k) \lambda(d k),
$$

and $\eta_{s}^{*}(d j)=\eta_{s}^{*}(j) \lambda(d j)$ is absolutely continuous with respect to $\lambda$, then

(iv) for all $t<u, \eta_{t}^{*}(d k)$ is absolutely continuous with respect to $\lambda$, with density

$$
\eta_{t}^{*}(k)=\int_{\mathbb{R}^{d}} \eta_{s}^{*}(j) h^{\dagger}(s, d j, t, k)=e^{-(t-s) H^{\dagger}} \eta_{s}^{*}(k), \quad r \leqslant s<t,
$$

(v) $\left(z_{t}\right)_{t \in[r, v]}$ is also a backward Markov process with transition kernel

$$
p^{*}(s, d j, t, k)=\frac{\eta_{s}^{*}(j)}{\eta_{t}^{*}(k)} h^{\dagger}(s, d j, t, k), \quad r \leqslant s<t,
$$


(vi) the law of $z_{t}$ at time $t$ is $\eta_{t}(k) \eta_{t}^{*}(k) \lambda(d k)$.

Proof. The fact $(i)$ that $p(s, j, t, d k)$ is a Markov transition kernel follows from the definition of $\eta_{t}(k)$ itself:

$$
\begin{aligned}
\int_{\mathbb{R}^{d}} p(t, k, u, d l) p(u, l, v, d m) & =\frac{\eta_{v}(m)}{\eta_{t}(k)} \int_{\mathbb{R}^{d}} h(t, k, u, d l) h(u, l, v, d m) \\
& =\frac{\eta_{v}(m)}{\eta_{t}(k)} h(t, k, v, d m)=p(t, k, v, d m) .
\end{aligned}
$$

The existence of the inhomogeneous Markov process $\left(z_{t}\right)_{t \in[r, v]}$ follows from e.g. Theorem 4.1.1 of [13] applied on the (complete separable) space $\mathbb{R}^{d}$. More precisely, [13] yields the existence of the space-time homogeneous Markov process $\left(t, z_{t}\right)_{t \in[r, v]}$ with transition semigroup

$$
\tilde{p}((t, k), s,(d u, d l))=p(t, k, u, d l) \delta_{t+s}(d u) .
$$

Let us show that (5.6) holds for this forward Markov process. We have, for $r \leqslant t_{1}<t_{2}<\cdots<t_{n} \leqslant v$,

$$
\begin{aligned}
& P\left(z_{t_{1}} \in d k_{1}, \ldots, z_{t_{n}} \in d k_{n}\right) \\
& \quad=\eta_{t_{1}}^{*}\left(d k_{1}\right) \eta_{t_{1}}\left(k_{1}\right) p\left(t_{1}, k_{1}, t_{2}, d k_{2}\right) \cdots p\left(t_{n-1}, k_{n-1}, t_{n}, d k_{n}\right) \\
& \quad=\eta_{t_{1}}^{*}\left(d k_{1}\right) h\left(t_{1}, k_{1}, t_{2}, d k_{2}\right) \cdots h\left(t_{n-1}, k_{n-1}, t_{n}, d k_{n}\right) \eta_{t_{n}}\left(k_{n}\right) .
\end{aligned}
$$

In particular, using (5.5),

$$
P\left(z_{s} \in d j, z_{u} \in d l\right)=\eta_{s}^{*}(d j) \eta_{s}(j) p(s, j, u, d l)=\eta_{s}^{*}(d j) h(s, j, u, d l) \eta_{u}(l),
$$

and

$$
P\left(z_{s} \in d j, z_{t} \in d k, z_{u} \in d l\right)=\eta_{s}^{*}(d j) h(s, j, t, d k) h(t, k, u, d l) \eta_{u}(l) .
$$

Hence $P\left(z_{t} \in d k \mid z_{s}=j, z_{u}=l\right)$ satisfies $\eta_{s}^{*}(d j)$-a.e.:

$$
P\left(z_{t} \in d k \mid z_{s}=j, z_{u}=l\right) h(s, j, u, d l)=h(s, j, t, d k) h(t, k, u, d l) .
$$

This gives, with $s_{1}<s_{2}<\cdots<s_{n}<t<u_{1}<\cdots<u_{m}$, and introducing the Bernstein kernel $h\left(s_{n}, j_{n}, t, A, u_{1}, l_{1}\right)$ $=P\left(z_{t} \in A \mid z_{s_{n}}=j_{n}, z_{u_{1}}=l_{1}\right)$ of Section 3,

$$
\begin{aligned}
P( & \left.z_{s_{1}} \in d j_{1}, \ldots, z_{s_{n}} \in d j_{n}, z_{t} \in A, z_{u_{1}} \in d l_{1}, \ldots, z_{u_{m}} \in d l_{m}\right) \\
= & \int_{A} \eta_{s_{1}}^{*}\left(d j_{1}\right) h\left(s_{1}, j_{1}, s_{2}, d j_{2}\right) \cdots h\left(s_{n}, j_{n}, t, d k\right) \\
& h\left(t, k, u_{1}, d l_{1}\right) \cdots h\left(u_{m-1}, l_{m-1}, u_{m}, d l_{m}\right) \eta_{u_{m}}\left(l_{m}\right) \\
= & h\left(s_{n}, j_{n}, t, A, u_{1}, l_{1}\right) \eta_{s_{1}}^{*}\left(d j_{1}\right) h\left(s_{1}, j_{1}, s_{2}, d j_{2}\right) \cdots h\left(s_{n-1}, j_{n-1}, s_{n}, d j_{n}\right) h\left(s_{n}, j_{n}, u_{1}, d l_{1}\right) \\
& h\left(u_{1}, l_{1}, u_{2}, d l_{2}\right) \cdots h\left(u_{m-1}, l_{m-1}, u_{m}, d l_{m}\right) \eta_{u_{m}}\left(l_{m}\right) \\
= & h\left(s_{n}, j_{n}, t, A, u_{1}, l_{1}\right) P\left(z_{s_{1}} \in d j_{1}, \ldots, z_{s_{n}} \in d j_{n}, z_{u_{1}} \in d l_{1}, \ldots, z_{u_{m}} \in d l_{m}\right),
\end{aligned}
$$

hence

$$
P\left(z_{t} \in d k \mid \mathcal{P}_{s_{n}} \vee \mathcal{F}_{u_{1}}\right)=h\left(s_{n}, z_{s_{n}}, t, d k, u_{1}, z_{u_{1}}\right)=P\left(z_{t} \in d k \mid z_{s_{n}}, z_{u_{1}}\right) .
$$

Finally, under the condition (5.7) we have

$$
\eta_{t}^{*}(d k)=\int_{\mathbb{R}^{d}} \eta_{s}^{*}(d j) h(s, j, t, d k)=\int_{\mathbb{R}^{d}} \eta_{s}^{*}(j) h(s, j, t, d k) \lambda(d j)=\int_{\mathbb{R}^{d}} \eta_{s}^{*}(j) h^{\dagger}(s, d j, t, k) \lambda(d k) .
$$


The process $\left(z_{t}\right)_{t \in[r, v]}$ being constructed from the forward kernel (3.2), we show that its backward kernel is given by (3.3) when (5.7) holds; we have

$$
\begin{aligned}
P & \left(z_{s} \in A, z_{t_{1}} \in d k_{1}, \ldots, z_{t_{n}} \in d k_{n}\right) \\
& =\int_{A} \eta_{s}^{*}(k) h^{\dagger}\left(s, d j, t_{1}, k_{1}\right) \cdots h^{\dagger}\left(t_{n-1}, d k_{n-1}, t_{n}, d k_{n}\right) \eta_{t_{n}}\left(d k_{n}\right) \\
& =\int_{A} p^{*}\left(s, d j, t_{1}, k_{1}\right) \cdots p^{*}\left(t_{n-1}, d k_{n-1}, t_{n}, k_{n}\right) \eta_{t_{n}}^{*}\left(d k_{n}\right) \eta_{t_{n}}\left(k_{n}\right) \\
& =p^{*}\left(s, A, t_{1}, k_{1}\right) p^{*}\left(t_{1}, d k_{1}, t_{2}, k_{2}\right) \cdots p^{*}\left(t_{n-1}, d k_{n-1}, t_{n}, k_{n},\right) \eta_{t_{n}}^{*}\left(d k_{n}\right) \eta_{t_{n}}\left(k_{n}\right) \\
& =p^{*}\left(s, A, t_{1}, k_{1}\right) P\left(z_{t_{1}} \in d k_{1}, \ldots, z_{t_{n}} \in d k_{n}\right),
\end{aligned}
$$

hence $\left(z_{t}\right)_{t \in[r, v]}$ is also backward Markovian with transition kernel $p^{*}\left(s, d j, t_{1}, k_{1}\right)$.

Relation (5.4) can be written as

$$
-\frac{\partial \eta_{t}^{*}(d k)}{\partial t}=H^{\dagger} \eta_{t}^{*}(d k) \quad \text { and } \quad \frac{\partial \eta_{t}}{\partial t}(k)=H \eta_{t}(k), \quad t \in[r, v] .
$$

The following similar proposition shows that Markovian bridges can also be constructed from backward Markov processes. Propositions 5.1 and 5.2 complete the proof of Theorem 3.2. Proposition 5.2 next is proved similarly to Proposition 5.1.

Proposition 5.2. Let $\eta_{r}^{*}: \mathbb{R}^{d} \rightarrow \mathbb{R}_{+}$and $\eta_{v}(d m)$ be initial and final conditions such that for some $t \in[r, v]$,

$$
\int_{\mathbb{R}^{d}} \eta_{t}^{*}(k) \eta_{t}(d k)=1
$$

where

$$
\eta_{t}(d k)=\int_{\mathbb{R}^{d}} \eta_{v}(d m) h^{\dagger}(t, d k, v, m), \quad \eta_{t}^{*}(k)=\int_{\mathbb{R}^{d}} \eta_{r}^{*}(i) h^{\dagger}(r, d i, t, k)=e^{-(t-r) H^{\dagger}} \eta_{r}^{*}(k),
$$

$r<t<v$, and

$$
p^{*}(s, d j, t, k)=\frac{\eta_{s}^{*}(j)}{\eta_{t}^{*}(k)} h^{\dagger}(s, d j, t, k) .
$$

Then

(i) $p^{*}(s, d j, t, k)$ is a backward Markov transition kernel,

(ii) the inhomogeneous backward Markov process $\left(z_{t}\right)_{t \in[s, u]}$ with transition kernel $p^{*}(s, d j, t, k)$ and final distribution $\eta_{u}(d l) \eta_{u}^{*}(l)$ satisfies

$$
P\left(z_{t} \in d k \mid \mathcal{P}_{s} \vee \mathcal{F}_{u}\right)=P\left(z_{t} \in d k \mid z_{s}, z_{u}\right),
$$

i.e. it is a Bernstein process.

(iii) the law at time $t$ of $z_{t}$ is $\rho_{t}(k)=\eta_{t}(k) \eta_{t}^{*}(d k)$.

If moreover $H$ and $H^{\dagger}$ are adjoint with respect to a fixed reference measure, i.e.

$$
h(t, k, u, d l) \lambda(d k)=h^{\dagger}(t, d k, u, l) \lambda(d l),
$$


and $\eta_{u}(d l)=\eta_{u}(l) \lambda(d l)$ is absolutely continuous with respect to $\lambda$, then

(iv) $\eta_{t}(d k)$ is absolutely continuous with respect to $\lambda$, with density

$$
\eta_{t}(k)=\int_{\mathbb{R}^{d}} \eta_{u}(l) h(t, k, u, d l)=e^{-(u-t) H} \eta_{u}(k), \quad t<u \leqslant v,
$$

(v) $\left(z_{t}\right)_{t \in[r, v]}$ is a forward Markov process with transition kernel

$$
p(t, k, u, d l)=\frac{\eta_{u}(l)}{\eta_{t}(k)} h(t, k, u, d l)
$$

(vi) the law at time $t$ of $z_{t}$ is $\eta_{t}(k) \eta_{t}^{*}(k) \lambda(d k)$.

Relation (5.9) can be written as

$$
-\frac{\partial \eta_{t}^{*}}{\partial t}(k)=H^{\dagger} \eta_{t}^{*}(k) \quad \text { and } \quad \frac{\partial \eta_{t}}{\partial t}(d k)=H \eta_{t}(d k) \quad t \in[r, v] .
$$

\section{Generators}

In this section we study the generators of Bernstein diffusions with jumps, solutions of forward and backward stochastic integro-differential equations, under the assumptions of Theorem 3.2.

Definition 6.1. For $f \in \mathcal{S}\left(\mathbb{R}^{d}\right)$ we define the forward generator (cf. Proposition 3.2) by

$$
\begin{aligned}
\mathcal{L}_{\eta_{t}} f(k)= & \langle c, \nabla f(k)\rangle+\frac{1}{2} \Delta_{B} f(k)+\int_{\mathbb{R}^{d}}\left(f(k+y)-f(k)-\langle y, \nabla f(k)| 1_{\{|y| \leqslant 1\}} \frac{\eta_{t}(k+y)}{\eta_{t}(k)} v(d y)\right. \\
& +\int_{\mathbb{R}^{d}} \frac{\eta_{t}(k+y)-\eta_{t}(k)}{\eta_{t}(k)}\langle y, \nabla f(k)| 1_{\{|y| \leqslant 1\}} v(d y)+\left\langle\nabla \log \eta_{t}(k), \nabla f(k)\right\rangle_{B},
\end{aligned}
$$

and its backward counterpart by

$$
\begin{aligned}
\mathcal{L}_{\eta_{t}^{*}}^{*} f(k)= & \langle c, \nabla f(k)\rangle-\frac{1}{2} \Delta_{B} f(k)-\int_{\mathbb{R}^{d}}\left(f(k-y)-f(k)+\langle y, \nabla f(k)\rangle 1_{\{|y| \leqslant 1\}}\right) \frac{\eta_{t}^{*}(k-y)}{\eta_{t}^{*}(k)} v(d y) \\
& +\int_{\mathbb{R}^{d}} \frac{\eta_{t}^{*}(k-y)-\eta_{t}^{*}(k)}{\eta_{t}^{*}(k)}\langle y, \nabla f(k)\rangle 1_{\{|y| \leqslant 1\}} v(d y)-\left\langle\nabla \log \eta_{t}^{*}(k), \nabla f(k)\right\rangle_{B} .
\end{aligned}
$$

Note that $\mathcal{L}_{\eta_{t}^{*}}^{*}$ is not the adjoint of $\mathcal{L}_{\eta_{t}}$, which will be denoted, when needed, by $\left(\mathcal{L}_{\eta_{t}}\right)^{\dagger}$. The proof of Proposition 3.4 follows from the next proposition.

Proposition 6.2. The kernels $p(t, k, u, d l)$ and $p^{*}(s, d j, t, k)$ of Propositions 5.1 and 5.2, satisfy the partial integrodifferential equations

$$
\frac{\partial p}{\partial u}(t, k, u, d l)=\left(\mathcal{L}_{\eta_{u}}\right)_{l}^{\dagger} p(t, k, u, d l)
$$

(Kolmogorov forward or Fokker-Planck equation), and

$$
\frac{\partial p^{*}}{\partial s}(s, d j, t, k)=\left(\mathcal{L}_{\eta_{s}^{*}}^{*}{ }_{j}^{\dagger} p(s, d j, t, k) .\right.
$$


The notation $\left(\mathcal{L}_{\eta_{u}}\right)_{l}^{\dagger} p(t, k, u, d l)$, respectively $\left(\mathcal{L}_{\eta_{s}^{*}}^{*}{ }_{j}^{\dagger} p(s, d j, t, k)\right.$, means that $\mathcal{L}_{\eta_{u}}$, respectively $\mathcal{L}_{\eta_{s}^{*}}^{*}$, acts on the variable $l$, respectively $k$, i.e. Proposition 6.2 states that

$$
\frac{\partial}{\partial u} \int_{\mathbb{R}^{d}} f(l) p(t, k, u, d l)=\int_{\mathbb{R}^{d}} \mathcal{L}_{\eta_{u}} f(l) p(t, k, u, d l),
$$

respectively

$$
\frac{\partial}{\partial s} \int_{\mathbb{R}^{d}} f(j) p^{*}(s, d j, t, k)=\int_{\mathbb{R}^{d}} \mathcal{L}_{\eta_{s}^{*}}^{*} f(j) p(s, d j, t, k) .
$$

In order to prove Proposition 6.2 we will need the following.

Lemma 6.3. For $f, g \in \mathcal{S}\left(\mathbb{R}^{d}\right)$, the carré du champ operators $[17,28]$ associated to $-H$ and $-H^{\dagger}$ are given respectively by

$$
\Gamma(f, g)(k)=U(k) f(k) g(k)+\langle\nabla f(k), \nabla g(k)\rangle_{B}+\int_{\mathbb{R}^{d}}(f(k+y)-f(k))(g(k+y)-g(k)) v(d y),
$$

and

$$
\Gamma^{\dagger}(f, g)(k)=U(k) f(k) g(k)+\langle\nabla f(k), \nabla g(k)\rangle_{B}+\int_{\mathbb{R}^{d}}(f(k-y)-f(k))(g(k-y)-g(k)) v(d y) .
$$

Proof. An elementary computation shows that

$$
-H(f g)=-f H g-g H f+\Gamma(f, g),
$$

and

$$
-H^{\dagger}(f g)=-f H g-g H f+\Gamma^{\dagger}(f, g),
$$

which is the definition of $\Gamma(f, g)$ and $\Gamma^{\dagger}(f, g)$.

Let the operators $D_{t}$ and $D_{t}^{*}$ be defined informally by

$$
D_{t} f=\frac{1}{\eta_{t}}\left(\frac{\partial}{\partial t}-H\right)\left(\eta_{t} f\right)
$$

and

$$
D_{t}^{*} f=\frac{1}{\eta_{t}^{*}}\left(\frac{\partial}{\partial t}+H^{\dagger}\right)\left(\eta_{t}^{*} f\right)
$$

By an adaptation of the method of [1] one shows that $D_{t}$ and $D_{t}^{*}$ are densely defined operators in $L^{2}\left(\mathbb{R}^{d}, \eta_{t}^{*}(k) \eta_{t}(k) \lambda(d k)\right)$. They will be called afterwards, the forward and backward derivatives, respectively.

The following lemma provides a decomposition of $D_{t}$ and $D_{t}^{*}$ which will be useful in the proof of Proposition 6.2.

Lemma 6.4. We have

$$
D_{t}=\frac{\partial}{\partial t}+\mathcal{L}_{\eta_{t}} \quad \text { and } \quad D_{t}^{*}=\frac{\partial}{\partial t}+\mathcal{L}_{\eta_{t}^{*}}^{*} .
$$

Proof. We have 


$$
\begin{aligned}
D_{t} f_{t}(k)= & \left(\frac{\partial}{\partial t}-H\right) f_{t}(k)+\frac{f_{t}(k)}{\eta_{t}(k)}\left(\frac{\partial}{\partial t}-H\right) \eta_{t}(k)+\frac{1}{\eta_{t}(k)} \Gamma\left(\eta_{t}, f_{t}\right)(k) \\
= & \frac{\partial f_{t}}{\partial t}(k)-V(i \nabla) f_{t}(k)+\left\langle\nabla \log \eta_{t}(k), \nabla f_{t}(k)\right\rangle_{B} \\
& +\int_{\mathbb{R}^{d}} \frac{\eta_{t}(k+y)-\eta_{t}(k)}{\eta_{t}(k)}\left(f_{t}(k+y)-f_{t}(k)\right) v(d y) \\
= & \frac{\partial f_{t}}{\partial t}(k)+\left\langle c, \nabla f_{t}(k)\right\rangle+\frac{1}{2} \Delta_{B} f_{t}(k)+\int_{\mathbb{R}^{d}}\left(f_{t}(k+y)-f_{t}(k)-\left\langle y, \nabla f_{t}(k)\right\rangle 1_{\{|y| \leqslant 1\}}\right) v(d y) \\
& +\left\langle\nabla \log \eta_{t}, \nabla f_{t}(k)\right\rangle_{B}+\int_{\mathbb{R}^{d}} \frac{\eta_{t}(k+y)-\eta_{t}(k)}{\eta_{t}(k)}\left(f_{t}(k+y)-f_{t}(k)\right) v(d y) \\
= & \frac{\partial f_{t}}{\partial t}(k)+\left\langle c, \nabla f_{t}(k)\right\rangle+\frac{1}{2} \Delta_{B} f_{t}(k) \\
& +\int_{\mathbb{R}^{d}}\left(\frac{\eta_{t}(k+y)}{\eta_{t}(k)}\left(f_{t}(k+y)-f_{t}(k)\right)-\left\langle y, \nabla f_{t}(k)\right\rangle 1_{\{|y| \leqslant 1\}}\right) v(d y)+\left\langle\nabla \log \eta_{t}, \nabla f_{t}(k)\right\rangle_{B} \\
= & \frac{\partial f_{t}}{\partial t}(k)+\left\langle c, \nabla f_{t}(k)\right\rangle+\left\langle\nabla \log \eta_{t}, \nabla f_{t}(k)\right\rangle_{B}+\frac{1}{2} \Delta_{B} f_{t}(k) \\
& +\int_{\mathbb{R}^{d}}\left(f_{t}(k+y)-f_{t}(k)-\left\langle y, \nabla f_{t}(k)\right| 1_{\{|y| \leqslant 1\}} \frac{\eta_{t}(k+y)}{\eta_{t}(k)} v(d y)\right. \\
& +\int_{\mathbb{R}^{d}} \frac{\eta_{t}(k+y)-\eta_{t}(k)}{\eta_{t}(k)}\left\langle y, \nabla f_{t}(k)\right\rangle 1_{\{|y| \leqslant 1\}} v(d y) \\
= & \left(\frac{\partial}{\partial t}+\mathcal{L}_{\eta_{t}}\right) f_{t}(k) .
\end{aligned}
$$

The proof for $D_{t}^{*}$ is similar.

Now we can easily prove Proposition 6.2.

Proof. We have for any $f \in \mathcal{S}\left(\mathbb{R}^{d}\right)$, using the decompositions of Lemma 6.4:

$$
\begin{aligned}
\eta_{t}(k) \frac{\partial}{\partial u} \int_{\mathbb{R}^{d}} f(l) p(t, k, u, d l) & =\frac{\partial}{\partial u} \int_{\mathbb{R}^{d}} f(l) \eta_{u}(l) h(t, k, u, d l)=\frac{\partial}{\partial u}\left[e^{-(u-t) H}\left(f \eta_{u}\right)(k)\right] \\
& =\int_{\mathbb{R}^{d}} f(l) \frac{\partial \eta_{u}}{\partial u}(l) h(t, k, u, d l)-\int_{\mathbb{R}^{d}} H f(l) \eta_{u}(l) h(t, k, u, d l) \\
& =\int_{\mathbb{R}^{d}} \eta_{t}(l) D_{u} f(l) h(t, k, u, d l)=\int_{\mathbb{R}^{d}} \eta_{u}(l) \mathcal{L}_{\eta_{u}} f(l) h(t, k, u, d l) \\
& =\eta_{t}(k) \int_{\mathbb{R}^{d}} \mathcal{L}_{\eta_{u}} f(l) p(t, k, u, d l)=\eta_{t}(k) \int_{\mathbb{R}^{d}} f(l)\left(\mathcal{L}_{\eta_{u}}\right)_{l}^{\dagger} p(t, k, u, d l) .
\end{aligned}
$$

The dual statement is proved similarly. 
Proof of Proposition 3.4. Proposition 6.2 shows that $f\left(z_{v}\right)-\int_{0}^{v} \mathcal{L}_{\eta_{u}} f\left(z_{u}\right) d u, v \geqslant 0$, is a martingale for $f \in \mathcal{S}\left(\mathbb{R}^{d}\right)$ :

$$
E\left[f\left(z_{v}\right)-f\left(z_{v}\right) \mid \mathcal{F}_{t}\right]=\int_{t}^{v} \frac{\partial}{\partial u} E\left[f\left(z_{u}\right) \mid \mathcal{F}_{t}\right] d u=\int_{t}^{v} E\left[\mathcal{L}_{\eta_{u}} f\left(z_{u}\right) \mid \mathcal{F}_{t}\right] d u,
$$

and $f\left(z_{v}\right)-\int_{0}^{v} \mathcal{L}_{\eta_{u}} f\left(z_{u}\right) d u, v \geqslant 0$, is a local martingale for $f \in \mathcal{C}^{2}\left(\mathbb{R}^{d}\right)$. A similar argument holds in the backward case.

\section{Uniqueness of reversible diffusions}

In this section we show that for the class of potentials considered in this paper the processes constructed are essentially the only Markovian reversible diffusions with jumps. As defined in Proposition 5.1 let us recall that, more generally, a Bernstein process is a process $\left(z_{t}\right)_{t \in[r, v]}$ such that

$$
P\left(z_{t} \in d k \mid \mathcal{P}_{s} \vee \mathcal{F}_{u}\right)=P\left(z_{t} \in d k \mid z_{s}, z_{u}\right), \quad r \leqslant s<t<u \leqslant v,
$$

where $\left(\mathcal{P}_{t}\right)_{t \in[r, v]}$, respectively $\left(\mathcal{F}_{t}\right)_{t \in[r, v]}$, denotes the increasing, respectively decreasing, filtration generated by

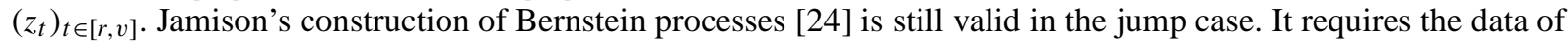
a probability measure $v$ on $\mathbb{R}^{d} \times \mathbb{R}^{d}$ and a Bernstein transition kernel, i.e. a kernel $h(s, j, t, d k, u, l)$ satisfying the counterpart of the Chapman-Kolmogorov equation:

$$
\int_{A} h(s, j, t, B, u, l) h(s, j, u, d l, v, m)=\int_{B} h(s, j, t, d k, v, m) h(t, k, u, A, v, m),
$$

for $A, B \in \mathcal{B}\left(\mathbb{R}^{d}\right)$. From [24] we know that there exists for these data a unique (in general not Markovian) Bernstein process $\left(z_{t}\right)_{t \in[r, v]}$ such that

(a) $P\left(z_{r} \in B, z_{v} \in C\right)=v(B \times C)$,

(b) $P\left(z_{t} \in B \mid z_{s}, z_{u}\right)=h\left(s, z_{s}, t, B, u, z_{u}\right), r \leqslant s<t<u \leqslant v$.

The finite dimensional distribution of $\left(z_{t}\right)_{t \in[r, v]}$ is given by

$$
\begin{aligned}
& P\left(z_{r} \in A, z_{t_{1}} \in B_{1}, \ldots, z_{t_{n}} \in B_{n}, z_{v} \in C\right) \\
& =\int_{A \times C} v(d j, d l) \int_{B_{1}} h\left(r, i, t_{1}, d k_{1}, v, m\right) \cdots \int_{B_{n}} h\left(t_{n-1}, k_{n-1}, t_{n}, d k_{n}, v, m\right),
\end{aligned}
$$

cf. [24].

Our construction of Markovian Bernstein processes did not follow, however, the above procedure. Instead, we started from the data of $U$ and $V$, defining $H=U+V(i \nabla)$ (Definition 2.1), i.e. from the Lévy process $\left(\xi_{t}\right)_{t \in[r, v]}$, and from boundary conditions $\eta_{r}^{*}$ and $\eta_{v}$, allowing to construct a Markov transition kernels with the solutions of the adjoint heat equations (3.4). Then we showed that the corresponding Markov process is a Bernstein process.

Conversely, under the additional hypothesis (7.4) and (7.5) on the kernel $h(s, j, t, d k, u, l)$ of a Bernstein process, it is possible to show that if a Bernstein process is Markovian then it is the process described in Theorem 3.2. This extends Theorem 3.1 of [24] and Theorem 3.3 of [31] to the case where $h(t, d k, u, d l)$ and $h^{\dagger}(s, d j, t, k)$ are not absolutely continuous with respect to a reference measure.

Theorem 7.1. Assume that $H$ and $H^{\dagger}$ are adjoint with respect to a measure $\lambda$. Then the conditions

$$
h(s, j, t, d k, u, l) h(s, j, u, d l)=h(s, j, t, d k) h(t, k, u, d l), \quad \lambda(d j) \text {-a.e., }
$$


and

$$
h(s, j, t, d k, u, l) h^{\dagger}(s, d j, u, l)=h^{\dagger}(s, d j, t, k) h^{\dagger}(t, d k, u, l), \quad \lambda(d l)-a . e .,
$$

are equivalent. Moreover,

(a) Let $\left(z_{t}\right)_{t \in[r, v]}$ denote the Bernstein process with kernel $h(s, j, t, d k, u, l)$ satisfying (7.4). Then the following are equivalent:

(i) the process $\left(z_{t}\right)_{t \in[r, v]}$ is forward Markovian and $p(t, k, u, d l)$ is absolutely continuous with respect to $h(t, k, u, d l)$,

(ii) there exists a measure $\eta_{r}^{*}(d i)$ and a positive density function $\eta_{v}(m)$ such that

$$
P\left(z_{r} \in A, z_{v} \in B\right)=\int_{A \times B} \eta_{r}^{*}(d i) h(r, i, v, d m) \eta_{v}(m) .
$$

(b) Assume that $h(s, j, t, d k, u, l)$ satisfies (7.5). Then the following are equivalent:

(iii) there exists a positive density function $\eta_{r}^{*}(i)$ and a probability measure $\eta_{v}(d m)$ such that

$$
P\left(z_{r} \in A, z_{v} \in B\right)=\int_{A \times B} \eta_{r}^{*}(i) h^{\dagger}(r, d i, v, m) \eta_{v}(d m) .
$$

(iv) the process $\left(z_{t}\right)_{t \in[r, v]}$ is backward Markovian and $p^{*}(s, d j, t, k)$ is absolutely continuous with respect to $h^{\dagger}(s, d j, t, k)$.

If $\eta_{r}^{*}(d i)=\eta_{r}^{*}(i) \lambda(d i)$ and $\eta_{v}(d l)=\eta_{v}(l) \lambda(d l)$ are absolutely continuous with respect to a fixed measure $\lambda$, then (i), (ii), (iii) and (iv) are equivalent.

Proof. Under the adjointness hypothesis of $H$ and $H^{\dagger}$ with respect to $\lambda$ :

$$
h^{\dagger}(s, d j, t, k) \lambda(d k)=h(u, j, t, d k) \lambda(d j),
$$

conditions (7.4) and (7.5) are equivalent since, then,

$$
h(s, j, t, d k, u, l) h(s, j, u, d l) \lambda(d j)=h(s, j, t, d k, u, l) h^{\dagger}(s, d j, u, l) \lambda(d l),
$$

and

$$
h(s, j, t, d k) h(t, k, u, d l) \lambda(d j)=h^{\dagger}(s, d j, t, k) \lambda(d l) h^{\dagger}(t, d k, u, l) .
$$

The implications (ii) $\Rightarrow$ (i), (iv) $\Rightarrow$ (iii) follows from Propositions 7.4, 7.5, and (i) $\Rightarrow$ (ii), (iii) $\Rightarrow$ (iv) will follow from Propositions 7.2, 7.3. Under the self-adjointness assumption (5.7), the equivalence (i) $\Leftrightarrow$ (iii) follows from Propositions 5.1 and 5.2, which show that the Bernstein process $\left(z_{t}\right)_{t \in[r, v]}$ is forward Markovian if and only if it is backward Markovian.

Proposition 7.2. Assume that the Bernstein kernel $h(s, j, t, d k, u, l)$ satisfies

$$
h(s, j, t, d k, u, l) h(s, j, u, d l)=h(s, j, t, d k) h(t, k, u, d l), \quad \rho_{s}(d j)-a . e .,
$$

where $\rho_{s}$ is the law of $z_{s}, r \leqslant s \leqslant v$. If the Bernstein process $\left(z_{t}\right)_{t \in[r, v]}$ is forward Markovian and $p(t, k, v, d m)$ is absolutely continuous with respect to $h(t, k, v, d m)$, then there exists a measure $\eta_{r}^{*}(d i)$ and a positive density function $\eta_{v}(m)$ such that

$$
P\left(z_{r} \in d i, z_{v} \in d m\right)=\eta_{r}^{*}(d i) h(r, i, v, d m) \eta_{v}(m), \quad r<v .
$$

Moreover we have

$$
p(r, i, t, d k)=\frac{\eta_{t}(k)}{\eta_{r}(i)} h(r, i, t, d k),
$$


with

$$
\eta_{t}(k)=\int_{\mathbb{R}^{d}} \eta_{v}(m) h(t, k, v, d m), \quad \eta_{t}^{*}(d k)=\int_{\mathbb{R}^{d}} \eta_{r}^{*}(d i) h(r, i, t, d k), \quad r \leqslant t \leqslant v .
$$

Proof. Let us assume that $\left(z_{t}\right)_{t \in[r, v]}$ is Markovian, with transition kernel $p(t, k, u, d l)$. Let $\rho_{r}(d i)$ denote an initial law of $\left(z_{t}\right)_{t \in[r, v] \text {. We have }}$

$$
P\left(z_{r} \in A, z_{t} \in B, z_{v} \in C\right)=\int_{A} \rho_{r}(d i) \int_{B} p(r, i, t, d k) \int_{C} p(t, k, v, d m) .
$$

On the other hand,

$$
P\left(z_{r} \in A, z_{t} \in B, z_{v} \in C\right)=\int_{A} \rho_{r}(d i) \int_{C} p(r, i, v, d m) \int_{B} h(r, i, t, d k, v, m) .
$$

Equating (7.10) and (7.11), we obtain

$$
p(r, i, t, d k) p(t, k, v, d m)=p(r, i, v, d m) h(r, i, t, d k, v, m),
$$

which, using (7.6), gives

$$
p(r, i, v, d m)=h(r, i, v, d m) \frac{p(r, i, t, d k)}{h(r, i, t, d k)} \frac{p(t, k, v, d m)}{h(t, k, v, d m)},
$$

and

$$
\begin{aligned}
v(A \times C) & =\int_{A} \rho_{r}(d i) \int_{C} p(r, i, v, d m) \\
& =\int_{A \times C} \rho_{r}(d i) \frac{p(r, i, t, d k)}{h(r, i, t, d k)} h(r, i, v, d m) \frac{p(t, k, v, d m)}{h(t, k, v, d m)}
\end{aligned}
$$

Let us fix $\left(t_{0}, k_{0}\right) \in \mathbb{R}_{+} \times \mathbb{R}^{d}$, and define

$$
\eta_{v}(m)=c\left(t_{0}, k_{0}\right) \frac{p\left(t_{0}, k_{0}, v, d m\right)}{h\left(t_{0}, k_{0}, v, d m\right)}
$$

and

$$
\eta_{r}^{*}(d i)=\frac{1}{c\left(t_{0}, k_{0}\right)} \frac{p\left(r, i, t_{0}, d k_{0}\right)}{h\left(r, i, t_{0}, d k_{0}\right)} \rho_{r}(d i),
$$

where $c\left(t_{0}, k_{0}\right)$ is a normalization constant equal to $\eta_{t_{0}}\left(k_{0}\right)$ after integrating in $d m$ the relation

$$
\eta_{v}(m) h\left(t_{0}, k_{0}, v, d m\right)=c\left(t_{0}, k_{0}\right) p\left(t_{0}, k_{0}, v, d m\right) .
$$

From (7.10), (7.12), (7.13) and (7.14) we have

$$
P\left(z_{r} \in d i, z_{v} \in d m\right)=\rho_{r}(d i) p(r, i, v, d m)=\eta_{r}^{*}(d i) h(r, i, v, d m) \eta_{v}(m),
$$

i.e. (7.7) holds. Finally, from (7.3) and (7.6) we have

$$
\begin{aligned}
P\left(z_{r} \in d i, z_{t} \in d k\right) & =\int_{\mathbb{R}^{d}} \eta_{r}^{*}(d i) h(r, i, v, d m) \eta_{v}(m) h(r, i, t, d k, v, m) \\
& =\int_{\mathbb{R}^{d}} \eta_{r}^{*}(d i) h(r, i, t, d k) h(t, k, v, d m) \eta_{v}(m) \\
& =\eta_{r}^{*}(d i) h(r, i, t, d k) \eta_{t}(k)
\end{aligned}
$$


and $P\left(z_{r} \in d i\right)=\eta_{r}^{*}(d i) \eta_{r}(i)$, which proves (7.8).

In the backward Markovian case we have the following result, which is proved similarly to Proposition 7.2.

Proposition 7.3. Assume that the Bernstein kernel $h(s, j, t, d k, u, l)$ satisfies

$$
h(s, j, t, d k, u, l) h^{\dagger}(s, d j, u, l)=h^{\dagger}(s, d j, t, k) h^{\dagger}(t, d k, u, l), \quad \rho_{u}(d l) \text {-a.e. },
$$

where $\rho_{u}$ is the law of $z_{u}, r \leqslant u \leqslant v$. If the Bernstein process $\left(z_{t}\right)_{t \in[r, v]}$ is backward Markovian and $p^{*}(r, d i, t, k)$ is absolutely continuous with respect to $h^{\dagger}(r, d i, t, k)$, then there exists a positive density function $\eta_{r}^{*}(i)$ and a measure $\eta_{v}(d m)$ such that

$$
P\left(z_{r} \in d i, z_{v} \in d m\right)=\eta_{r}(i) h^{\dagger}(r, d i, v, m) \eta_{v}^{*}(d m) .
$$

Moreover we have

$$
p^{*}(t, d k, v, m)=\frac{\eta_{t}^{*}(k)}{\eta_{v}^{*}(m)} h^{\dagger}(t, d k, v, m),
$$

with

$$
\eta_{t}(d k)=\int_{\mathbb{R}^{d}} \eta_{v}(d m) h^{\dagger}(t, d k, v, m), \quad \eta_{t}^{*}(k)=\int_{\mathbb{R}^{d}} \eta_{r}^{*}(i) h^{\dagger}(r, d i, t, k), \quad r \leqslant t \leqslant v .
$$

The following is a converse to Proposition 7.2.

Proposition 7.4. Assume that there exists a measure $\eta_{r}^{*}(d i)$ and a positive density function $\eta_{v}(m)$ such that

$$
v(A \times B)=P\left(z_{r} \in A, z_{v} \in B\right)=\int_{A \times B} \eta_{r}^{*}(d i) h(r, i, v, d m) \eta_{v}(m) .
$$

Then the Bernstein process $\left(z_{t}\right)_{t \in[r, v]}$ with kernel $h(s, j, t, d k, u, l)$ satisfying the conditions (7.4) or (7.5) is forward Markovian and $p(t, k, u, d l)$ is absolutely continuous with respect to $h(t, k, u, d l)$, and given by (7.8).

Proof. From (7.3), (7.4) and (7.17) we have

$$
\begin{aligned}
& P\left(z_{t_{1}} \in d k_{1}, \ldots, z_{t_{n}} \in d k_{n}, z_{u} \in d l\right) \\
& \quad=\int_{\mathbb{R}^{d}} \eta_{r}^{*}(d i) h\left(r, i, t_{1}, d k_{1}\right) \cdots h\left(t_{n}, k_{n}, u, d l\right) \int_{\mathbb{R}^{d}} \eta_{v}(m) h(u, l, v, d m) \\
& \quad=P\left(z_{t_{1}} \in d k_{1}, \ldots, z_{t_{n}} \in d k_{n}\right) h\left(t_{n}, k_{n}, u, d l\right) \frac{\int_{\mathbb{R}^{d}} \eta_{v}(m) h(u, l, v, d m)}{\int_{\mathbb{R}^{d}} \eta_{v}(m) h\left(t_{n}, k_{n}, v, d m\right)},
\end{aligned}
$$

hence

$$
p\left(t_{n}, k_{n}, u, d l\right)=\frac{\int_{\mathbb{R}^{d}} \eta_{v}(m) h(u, l, v, d m)}{\int_{\mathbb{R}^{d}} \eta_{v}(m) h\left(t_{n}, k_{n}, v, d m\right)} h\left(t_{n}, k_{n}, u, d l\right) .
$$

Of course, from (7.3), (7.5) and (7.18), it is also true that

Proposition 7.5. Assume that there exists a positive density function $\eta_{r}^{*}(i)$ and a measure $\eta_{v}(d m)$ such that

$$
P\left(z_{r} \in A, z_{v} \in B\right)=\int_{A \times B} \eta_{r}^{*}(i) h^{\dagger}(r, d i, v, m) \eta_{v}(d m) .
$$


Then the Bernstein process $\left(z_{t}\right)_{t \in[r, v]}$ with kernel $h(s, j, t, d k, u, l)$ satisfying (7.4) or (7.5) is backward Markovian and $p^{*}(s, d j, t, k)$ is absolutely continuous with respect to $h^{\dagger}(s, d j, t, k)$, and given by (7.16).

\section{Variational characterization}

In this section we use the approach to stochastic control for jump processes of [15,29], to obtain a variational characterization of the Markovian Bernstein processes (or reversible diffusions) with jumps considered before. We consider the stochastic control problem $\inf _{f} J(t, k ; f)$ with action functional

$$
J(t, k ; f)=E_{(t, k)}\left[\int_{t}^{v} L\left(z(s), f_{s}\right) d s-\log \eta_{v}(z(v))\right],
$$

where $E_{(t, k)}$ denotes the conditional expectation given $\left\{z_{t}=k\right\}$, and the Lagrangian $L(k, f)$ is defined informally as

$$
L(k, f)=\mathcal{L}_{f} \log f(k)+\frac{1}{f(k)} H f(k), \quad f \in \mathcal{S}\left(\mathbb{R}^{d}\right), f>0,
$$

where $\mathcal{L}_{f}$ is defined at the beginning of Section 3.2. We have explicitly

$$
\begin{aligned}
L(k, f) & =\int_{\mathbb{R}^{d}}\left(\frac{f(k)-f(k+y)}{f(k)}+\frac{f(k+y)}{f(k)} \log \frac{f(k+y)}{f(k)}\right) v(d y)+\frac{1}{2}\langle\nabla \log f(k), \nabla \log f(k)\rangle_{B}+U(k) \\
& =\int_{\mathbb{R}^{d}} g\left(\frac{\delta f(k, y)}{f(k)}\right) v(d y)+\frac{1}{2}\langle\nabla \log f(k), \nabla \log f(k)\rangle_{B}+U(k),
\end{aligned}
$$

with $g(x)=(1+x) \log (1+x)-x$ and $\delta f(k, y)=f(k+y)-f(k)$. In particular, when $f=\eta_{t}$,

$$
\begin{aligned}
L\left(k, \eta_{t}\right) & =\mathcal{L}_{\eta_{t}} \log \eta_{t}(k)+\frac{1}{\eta_{t}(k)} H \eta_{t}(k) \\
& =\mathcal{L}_{\eta_{t}} \log \eta_{t}(k)+\frac{1}{\eta_{t}(k)} \frac{\partial}{\partial t} \eta_{t}(k) \\
& =\mathcal{L}_{\eta_{t}} \log \eta_{t}(k)+\frac{\partial}{\partial t} \log \eta_{t}(k) \\
& =D_{t} \log \eta_{t}(k) .
\end{aligned}
$$

Proposition 8.1. The dynamic programming equation with final boundary condition

$$
\frac{\partial A_{t}}{\partial t}(k)+\min _{f}\left[\mathcal{L}_{f} A_{t}(k)+L\left(k, f_{t}\right)\right]=0, \quad A_{v}=-\log \eta_{v},
$$

associated to the action functional (8.1) has the solution $A_{t}=-\log \eta_{t}$, the minimum in $f$ being attained on $f_{t}(k)=\eta_{t}(k)$, i.e. when $A_{t}$ is solution of the Hamilton-Jacobi-Bellman equation

$$
\begin{aligned}
\frac{\partial A_{t}}{\partial t}(k)= & -U(k)-\frac{1}{2}\left(\Delta_{B} A_{t}(k)-\left\langle\nabla A_{t}(k), \nabla A_{t}(k)\right\rangle_{B}\right) \\
& +\int_{\mathbb{R}^{d}}\left(e^{-A_{t}(k+y)+A_{t}(k)}-1+1_{\{|y| \leqslant 1\}}\left\langle y, \nabla A_{t}(k)\right\rangle\right) d \nu(y)-\left\langle c, \nabla A_{t}(k)\right\rangle .
\end{aligned}
$$


Proof. We first show that for $g_{t}(k)>0$ :

$$
\min _{f}\left[-\mathcal{L}_{f_{t}} \log g_{t}(k)+L\left(k, f_{t}\right)\right]=\frac{1}{g_{t}(k)} H g_{t}(k),
$$

and that the minimum is attained for $f_{t}=g_{t}$. Let us define

$$
F(k, y)=-\frac{f_{t}(k+y)}{f_{t}(k)} \log \frac{g_{t}(k+y)}{g_{t}(k)}+\frac{f_{t}(k+y)}{f_{t}(k)} \log \frac{f_{t}(k+y)}{f_{t}(k)}-\frac{f_{t}(k+y)}{f_{t}(k)}+\frac{g_{t}(k+y)}{g_{t}(k)} .
$$

We have

$$
\begin{aligned}
& L\left(k, f_{t}\right)-\mathcal{L}_{f_{t}} \log g_{t}(k)-\frac{1}{\eta_{t}(k)} H \eta_{t}(k) \\
& \quad=-\mathcal{L}_{f_{t}} \log \frac{g_{t}(k)}{f_{t}(k)}+\frac{1}{f_{t}(k)} H f_{t}(k)-\frac{1}{\eta_{t}(k)} H \eta_{t}(k) \\
& \quad=\int_{\mathbb{R}^{d}} F(k, y) v(d y)+\frac{1}{2}\left\langle\frac{\nabla g_{t}}{g_{t}}(k)-\frac{\nabla f_{t}}{f_{t}}(k), \frac{\nabla g_{t}}{g_{t}}(k)-\frac{\nabla f_{t}}{f_{t}}(k)\right\rangle_{B} \\
& \quad \geqslant \int_{\mathbb{R}^{d}} F(k, y) v(d y) .
\end{aligned}
$$

Now, for all $a>0$,

$$
\min _{z \in \mathbb{R}}\left(z a+a \log a-a+e^{-z}\right)=0,
$$

hence taking $z=-\log \left(g_{t}(k+y) / g_{t}(k)\right)$ and $a=f_{t}(k+y) / f_{t}(k)$, we have $F(k, y) \geqslant 0$, and

$$
L\left(k, f_{t}\right)-\mathcal{L}_{f_{t}} \log g_{t}-\frac{1}{g_{t}} H g_{t}=-\mathcal{L}_{f_{t}} \log \frac{g_{t}}{f_{t}}+\frac{1}{f_{t}} H f_{t}-\frac{1}{g_{t}} H g_{t} \geqslant 0 .
$$

The minimum (zero) being attained with $f=g_{t}$, i.e.:

$$
\min _{f}\left[L\left(k, f_{t}\right)-\mathcal{L}_{f_{t}} \log g_{t}\right]=\frac{1}{g_{t}} H g_{t} .
$$

Letting $A_{t}=-\log g_{t}$, the dynamic programming equation (8.2) becomes

$$
\frac{\partial A_{t}}{\partial t}+e^{A_{t}} H e^{-A_{t}}=0
$$

with solution $A_{t}=-\log \eta_{t}$. Finally, from the relation

$$
\Delta_{B} A_{t}=-\frac{1}{g_{t}} \Delta_{B} g_{t}+\left\langle\frac{\nabla g_{t}}{g_{t}}, \frac{\nabla g_{t}}{g_{t}}\right\rangle_{B},
$$

we have

$$
\begin{aligned}
\frac{1}{g_{t}(k)} H g_{t}(k)= & \frac{1}{g_{t}(k)}\left(U(k) g_{t}(k)-\left\langle c, \nabla g_{t}(k)\right\rangle-\frac{1}{2} \Delta_{B} g_{t}(k)\right. \\
& \left.-\int_{\mathbb{R}^{d}}\left(g_{t}(k+y)-g_{t}(k)-\left\langle y, \nabla g_{t}(k)\right\rangle 1_{\{|y| \leqslant 1\}}\right) v(d y)\right) \\
= & U(k)+\left\langle c, \nabla A_{t}(k)\right\rangle+\frac{1}{2}\left(\Delta_{B} A_{t}-\left\langle\nabla A_{t}, \nabla A_{t}\right\rangle_{B}\right) \\
& -\int_{\mathbb{R}^{d}}\left(e^{-A_{t}(k+y)+A_{t}(k)}-1+1_{\{|y| \leqslant 1\}}\left\langle y, \nabla A_{t}\right\rangle\right) d \nu,
\end{aligned}
$$


which yields (8.3).

In the backward case we consider the action functional which is, informally, the time reversed of (8.1):

$$
J^{*}\left(t, k ; f^{*}\right)=E_{(t, k)}\left[\int_{r}^{t} L^{*}\left(z(s), f^{*}\right) d s-\log \eta_{r}^{*}(z(r))\right]
$$

with Lagrangian $L^{*}\left(k, f^{*}\right)$ defined now as

$$
L^{*}\left(k, f^{*}\right)=-\mathcal{L}_{f^{*}}^{*} \log f^{*}(k)+\frac{1}{f^{*}(k)} H^{\dagger} f^{*}(k), \quad f^{*} \in \mathcal{S}\left(\mathbb{R}^{d}\right) .
$$

We have

$$
L^{*}\left(k, f^{*}\right)=\int_{\mathbb{R}^{d}} g\left(\frac{\delta^{*} f^{*}(k, y)}{f^{*}(k)}\right) v(d y)+\frac{1}{2}\left\langle\nabla \log f^{*}(k), \nabla \log f^{*}(k)\right\rangle_{B}+U(k),
$$

with $\delta^{*} f^{*}(k, y)=f^{*}(k-y)-f^{*}(k)$. In particular,

$$
L^{*}\left(k, \eta_{t}^{*}\right)=-D_{t}^{*} \log \eta_{t}^{*}(k)
$$

Similarly we have the following proposition.

Proposition 8.2. The backward dynamic programming equation with initial boundary condition

$$
\frac{\partial A_{t}^{*}}{\partial t}(k)+\min _{f^{*}}\left[-\mathcal{L}_{f^{*}}^{*} A_{t}^{*}(k)+L\left(k, f_{t}^{*}\right)\right]=0, \quad A_{r}^{*}=-\log \eta_{r}^{*},
$$

associated to (8.5) has solution $A_{t}^{*}=-\log \eta_{t}^{*}$, the minimum in $f^{*}$ being attained at $f_{t}^{*}(k)=\eta_{t}^{*}(k)$, and $A_{t}^{*}$ is solution of the backward Hamilton-Jacobi-Bellman equation

$$
\begin{aligned}
\frac{\partial A_{t}^{*}}{\partial t}(k)= & U(k)+\frac{1}{2}\left(\Delta_{B} A_{t}^{*}(k)-\left\langle\nabla A_{t}^{*}(k), \nabla A_{t}^{*}(k)\right\rangle_{B}\right) \\
& -\int_{\mathbb{R}^{d}}\left(e^{-A_{t}^{*}(k-y)+A_{t}^{*}(k)}-1-1_{\{|y| \leqslant 1\}}\left\langle y, \nabla A_{t}^{*}(k)\right\rangle\right) d v(y)-\left\langle c, \nabla A_{t}^{*}(k)\right\rangle .
\end{aligned}
$$

In summary, we have shown here that the diffusion processes with jumps constructed before can also be regarded as minima of some stochastic action functionals associated with the starting $H$.

\section{Acknowledgements}

This work has been initiated during a visit of the first author to the GFM (Group of Mathematical Physics) under a "Programatico" Grant conceded by the FCT with the support of the POCTI Project POCTI/MAT/34924/2000, and funded in cooperation with the European Community Fund FEDER. The authors are also thankful for the support of the franco-portuguese program “GRICES-Ambassade de France au Portugal” No 631 B2.

\section{References}

[1] S. Albeverio, J. Rezende, J.-C. Zambrini, Probability and quantum symmetries II. The theorem of Noether in quantum mechanics, submitted for publication. 
[2] S. Albeverio, K. Yasue, J.-C. Zambrini, Euclidean quantum mechanics: analytical approach, Ann. Inst. H. Poincaré Phys. Théor. 50 (3) (1989) 259-308.

[3] S. Bernstein, Sur les liaisons entre les grandeurs aléatoires, in: Verh. des intern. Mathematikerkongr., vol. 1, 1932, Zürich.

[4] J. Bertoin, Lévy Processes, in: Cambridge Tracts in Mathematics, vol. 121, Cambridge Univ. Press, 1998.

[5] A. Beurling, An automorphism of product measures, Ann. of Math. (2) 72 (1960) 189-200.

[6] P. Cattiaux, Time reversal of diffusion processes with a boundary condition, Stochastic Process. Appl. 28 (2) (1988) 275-292.

[7] P. Cattiaux, C. Léonard, Large deviations and Nelson processes, Forum Math. 7 (1) (1995) 95-115.

[8] K.L. Chung, J.-C. Zambrini, Introduction to Random Times and Quantum Randomness, new edition, World Scientific, 2003.

[9] A.B. Cruzeiro, L. Wu, J.-C. Zambrini, Bernstein processes associated with a Markov process, in: Stochastic Analysis and Mathematical Physics (Santiago, 1998), Birkhäuser Boston, Boston, MA, 2000, pp. 41-72.

[10] R.L. Dobrushin, Yu.M. Sukhov, I. Fritts, A.N. Kolmogorov—founder of the theory of reversible Markov processes, Uspekhi Mat. Nauk. 43 (6(264)) (1988) 167-188.

[11] J.L. Doob, Classical Potential Theory and its Probabilistic Counterpart, Springer-Verlag, Berlin, 1984.

[12] M. Emery, M. Yor, A parallel between Brownian bridges and gamma bridges, Prépublication No 846, Laboratoire de Probabilités de l’Université Paris VI, 2003.

[13] S.N. Ethier, T.G. Kurtz, Markov Processes, Characterization and Convergence, Wiley, New York, 1986.

[14] P. Fitzsimmons, J. Pitman, M. Yor, Markovian bridges: Construction, Palm interpretation, and splicing, in: E. Cinlar, et al. (Eds.), Seminar on Stochastic Processes, in: Prog. Probab., vol. 33, Birkhäuser, Washington, 1992, pp. 101-134.

[15] W.H. Fleming, H.M. Soner, Controlled Markov Processes and Viscosity Solutions, Springer-Verlag, New York, 1993.

[16] J.M. Hammersley, Harnesses, in: Proc. Fifth Berkeley Sympos. Mathematical Statistics and Probability (Berkeley, Calif., 1965/66), vol. III: Physical Sciences, Univ. California Press, Berkeley, CA, 1967, pp. 89-117.

[17] F. Hirsch, Opérateurs carré du champ (d'après J.P. Roth), in: Séminaire Bourbaki, 29e année (1976/77), in: Lecture Notes in Math., vol. 677, Springer, Berlin, 1978, pp. 167-182.

[18] G.A. Hunt, Markoff chains and Martin boundaries, Illinois J. Math. 4 (1960) 313-340.

[19] T. Ichinose, S. Takanobu, The norm estimate of the difference between the Kac operator and Schrödinger semigroup. II. The general case including the relativistic case, Electron. J. Probab. 5 (4) (2000) 47 (electronic).

[20] N. Ikeda, S. Watanabe, Stochastic Differential Equations and Diffusion Processes, North-Holland, Amsterdam, 1989.

[21] N. Jacob, R.L. Schilling, Lévy-type processes and pseudodifferential operators, in: S. Resnick, O. Barndorff-Nielsen, Th. Mikosch (Eds.), Lévy Processes, Birkhäuser Boston, Boston, MA, 2001, pp. 139-168.

[22] J. Jacod, Calcul stochastique et problèmes de martingales, in: Lecture Notes in Mathematics, vol. 714, Springer-Verlag, Berlin, 1979.

[23] J. Jacod, P. Protter, Time reversal on Lévy processes, Ann. Probab. 16 (2) (1988) 620-641.

[24] B. Jamison, Reciprocal processes, Z. Wahr. Verw. Geb. 30 (1974) 65-86.

[25] T. Komatsu, Markov processes associated with certain integro-differential operators, Osaka J. Math. 10 (1973) 271-303.

[26] J.-P. Lepeltier, B. Marchal, Problème des martingales et équations différentielles stochastiques associées à un opérateur intégro-différentiel, Ann. Inst. H. Poincaré Sect. B (N.S.) 12 (1) (1976) 43-103.

[27] N. Privault, J.-C. Zambrini, Euclidean quantum mechanics in the momentum representation, 2004, in preparation.

[28] J.P. Roth, Opérateurs carré du champ et formule de Lévy-Kinchine sur les espaces localement compacts, C. R. Acad. Sci. Paris Sér. A 278 (1974) 1103-1106.

[29] S.J. Sheu, Stochastic control and exit probabilities of jump processes, SIAM J. Control Optim. 23 (2) (1985) 306-328.

[30] D. Stroock, Diffusion processes associated with Lévy generators, Z. Wahr. Verw. Geb. 32 (3) (1975) 209-244.

[31] J.-C. Zambrini, Variational processes and stochastic versions of mechanics, J. Math. Phys. 27 (9) (1986) 2307-2330. 